\title{
Crocidura (Mammalia, Soricidae) remains from the late Early Pleistocene Somssich Hill 2 locality (Villány Hills, Southern Hungary)
}

\author{
Dániel Bot KA \& Lukács MÉszÁRos \\ Department of Palaeontology, Eötvös Loránd University, \\ H-1117 Budapest, Pázmány Péter sétány 1/C, Hungary. \\ E-mail:botkadani@gmail.com,lgy.meszaros@gmail.com
}

\begin{abstract}
Two species of the white-toothed shrews (Crocidura kornfeldi Kormos, 1934 and Crocidura obtusa Kretzoi, 1938) were found in the late Early Pleistocene vertebrate fauna of the Somssich Hill 2 locality. Most of the specimens could not be identified at the species level, therefore they are described here as Crocidura sp. indet. (kornfeldi or obtusa). Detailed morphological and morphometrical analyses were made on the remains of the two species. The biostratigraphical and palaeoecological relations of their occurrence are also given. With 14 figures and 5 tables.
\end{abstract}

Key words - Crocidura kornfeldi, Crocidura obtusa, Early Pleistocene, Somssich Hill, Soricidae

\section{INTRODUCTION}

The Somssich Hill 2 site is one of the most important Pleistocene vertebrate localities of Hungary. It was rediscovered by Dénes Jánossy and György Topál in 1974 (it was already mentioned by Kormos (1937) and Kretzor (1956)). The excavation led by them between 1975 and 1984 yielded rich Pleistocene fauna, the preliminary lists of which are given in JÁnOSsY $(1983,1986,1990)$. The molluscan fauna of the locality was elaborated by KroLOPP (2000), and some mammal groups were also described by János Hír (cricetids) and Dénes Jánossy (lemmings and arvicolids) (Hír 1998, JÁnossy 1983, 1990). Most of the Somssich Hill 2 material is under elaboration in the Department of Palaeontology and Geology of the Hungarian Natural History Museum, by the cooperative research group of the Hungarian Academy of Sciences, the Hungarian Natural History Museum and the Eötvös Loránd University (OTKA K104506, project leader: Piroska Pazonyi). Some articles were recently published on the results of some fossil groups of the locality (BotKa \& MÉszÁros 2014a, 2015, STRICZKY \& Pazonyi 2014, SZentesi 2014).

A great number of soricid fossils were found in the Somssich Hill 2 assemblage. Seven shrew species were mentioned by BotKA \& MÉszÁros (2014b) 
and MÉszáros (2015). In the first description of the locality, JÁnOSSY (1983) mentioned only one Crocidura species (C. obtusa). In the present study, we determined two species of this genus (C. kornfeldi and C. obtusa), which are discussed here in detail.

\section{LOCALITY}

The Somssich Hill 2 site is located ca. 500 m west from the city of Villány, on the top of the Somssich Hill (today named as Villány Hill) in Southern Hungary, Villány Hills (GPS coordinates: N $45^{\circ} 52^{\prime} 26.66^{\prime \prime}$, E 18 $26^{\prime} 32.71$ ”; EOVX = 58998, EOVY = 603025).

Detailed description of the locality is given in Hungarian by BOTKA \& MÉszÁros (2015). Brief English overviews are shown in BOTKA \& MÉszÁros (2014a) and in STRICZKY \& PAZONYi (2014).

\section{MATERIAL AND METHODS}

The fossil material of the locality is stored in the Department of Palaeontology and Geology of the Hungarian Natural History Museum, Budapest (inventory numbers of the studied white-toothed shrews: VER 2015.247.-364.; VER 2015.305.-335.). BоTKA \& MÉszÁros (2014a) mentioned that the Beremendia remains are poorly preserved, they are fragmentary and complete mandibles and maxillae are relatively rare. The Crocidura material is even less wellpreserved, most of the remains are isolated teeth.

The 50 layers yielded 208 Crocidura remains, 12 specimens of which could have been identified with certainty as C. kornfeldi and 15 as C. obtusa. 181 specimens (isolated teeth, maxillary or mandible fragments) were not exactly identifiable, thus they are described as Crocidura sp. indet. (kornfeldi or obtusa). The presence of a third Crocidura species in the material is probably to be excluded.

During anatomical descriptions and measurements the method presented by REUMER (1984) was followed. The measurements were taken using the Delta Optical Smart Analysis Pro 1.0.0 software and were given in $\mathrm{mm}$. The SEM photos were taken by a Hitachi S-2360N Environmental Scanning Electron Microscope (ESEM).

The abbreviations in the descriptions and in the measurements are used as follows: $\mathrm{I}=$ incisor, $\mathrm{A}=$ antemolar, $\mathrm{P}=$ premolar, $\mathrm{M}=$ molar, $\mathrm{M}^{\mathrm{x}}=$ upper tooth, $\mathrm{M}_{\mathrm{x}}=$ lower tooth, $\mathrm{L}=$ length, $\mathrm{W}=$ width, $\mathrm{H}=$ height, $\mathrm{BL}=$ buccal length, $\mathrm{LL}$ = lingual length, $\mathrm{AW}=$ anterior width, $\mathrm{PW}=$ posterior width, $\mathrm{n}=$ number of specimens, $\min .=$ minimum, $\max .=$ maximum, $S D=$ standard deviation, $\mathrm{MNI}$ $=$ minimum number of individuals, $\mathrm{GMH}=$ Geological Museum of Hungary (in 
the Hungarian Geological and Geophysical Institute). The numbers in the lists of "Studied material and measurements" are the collection and working numbers used during the elaboration of the material.

\section{SYSTEMATIC DESCRIPTION}

Phylum Vertebrata Linnaeus, 1758

Classis Mammalia Linnaeus, 1758

Order Eulipotyphla Waddell et al., 1999

Family Soricidae Fischer von Waldheim, 1817

Subfamily Crocidurinae Milne-Edwards, 1874

Genus Crocidura Wagler, 1832

Dental formula is $143 / 123$. The dental elements are not stained. The lower incisor is short to moderately long and is curving upward, its cutting edge is slightly serrate to smooth. The first upper antemolar is larger than the equal-sized second and third antemolars, all of them are unicuspid (after REPENNING 1967). The genus is present in the European mainland from the Early Villányian ( $\mathrm{MN}$ 16 zone, Pliocene) to present.

\section{Crocidura kornfeldi Kormos, 1934}

(Figs 1-2)

1934 Crocidura kornfeldi n. sp. - Kormos, pp. 304-305, fig. 37.

1983 Crocidura kornfeldi Kormos - FEJFAR \& HoRÁČEK, pp. 134-136, tab. 2, pl. VI, figs 1-10, pl.

VII, figs 7-10.

1984 Crocidura kornfeldi Kormos - Reumer, pp. 18-22, tabs 1-2, pl. 1, figs 1-6, pl. 2, figs 1-5.

1985 Crocidura kornfeldi Kormos - REUMER \& DoukAs, pp. 114-115, pl. 2, fig. 1.

1985 Crocidura sp. - GIL \& SESÉ, pp. 495-496, fig. 1.

2000 Crocidura kornfeldi Kormos - RzeBIK-KowalskA, pp. 36-39, tabs XXXI-XXXIII, figs 13A, 14.

2001 Crocidura kornfeldi Kormos - Koufos et al., pp. 53-55, tabs 3-4, fig. 2, pl. 2, figs 13a-22b.

2005 Crocidura kornfeldi Kormos - MASINI et al., pp. 82-83, tab. 1, fig. 4: 2-6.

2011 Crocidura kornfeldi Kormos - Rofes \& CuenCA-Bescós, pp. 66-70, tabs 1-2, figs 2-3.

2013 Crocidura kornfeldi Kormos - CuencA-Bescós et al., fig. 4G.

2015 Crocidura kornfeldi Kormos - Furió et al., p. 156, tab. 2, figs 3, 5a-b.

Holotype - A skull with partial dentition, GMH Ob. 3686.

Type locality - Villány 3 locality, Villány Hills, Hungary, Early Pleistocene (Villányian Stage, Kisláng Phase).

Studied material and measurements - For the overview of the measurements see Table 1 . The measurement data of the following list are given in $\mathrm{mm}$. 


\section{Layer 5 (VER 2015.247.)}

2.5/12 - Right mandible fragment with $\mathrm{M}_{2}$ and $\mathrm{M}_{3} ; \mathrm{M}_{2}: \mathrm{L}=1.620, \mathrm{~W}=1.026 ; \mathrm{M}_{3}: \mathrm{L}=1.269, \mathrm{~W}=0.675$ 2.5/54, 55 - 2 right mandible fragments

2.5/71 - Right mandible fragment with $\mathrm{M}_{2}$ and $\mathrm{M}_{3} ; \mathrm{M}_{2}: \mathrm{L}=1.566, \mathrm{~W}=1.161 ; \mathrm{M}_{3}: \mathrm{L}=1.404, \mathrm{~W}=0.729$

\section{Layer 8 (VER 2015.248.)}

2.8/4 - Left mandible fragment

Layer 12 (VER 2015.249.)

$2.12 / 2$ - Right mandible fragment with $M_{2}$ and $M_{3} ; M_{2}: L=1.242, W=0.942 ; M_{3}: L=1.026, W=0.648$ Layer 14 (VER 2015.250.)

2.14/1 - Left mandible fragment with $\mathrm{M}_{2}$ and $\mathrm{M}_{3} ; \mathrm{M}_{2}: \mathrm{L}=1.485, \mathrm{~W}=1.031 ; \mathrm{M}_{3}: \mathrm{L}=1.161, \mathrm{~W}=0.675$

\section{Layer 30 (VER 2015.251.)}

2.30/2 - Left mandible fragment with $A_{2}, M_{1}$ and $M_{2} ; M_{1}: L=1.544, W=1.296 ; M_{2}: L=1.525$, $\mathrm{W}=1.004$

2.30/3 - Left mandible fragment with $M_{1}, M_{2}$ and $M_{3} ; M_{1}: L=1.647, W=1.209 ; M_{2}: L=1.539$, $\mathrm{W}=1.080 ; \mathrm{M}_{3}: \mathrm{L}=1.269, \mathrm{~W}=0.661$

\section{Layer 31 (VER 2015.252.)}

$2.31 / 2$ - Right mandible fragment

Layer 45 (VER 2015.253.)

2.45/1 - Right mandible fragment

\section{Layer 46 (VER 2015.254.)}

$2.46 / 3$ - Right mandible with $\mathrm{M}_{1} ; \mathrm{L}=1.606, \mathrm{~W}=1.142$

Original diagnosis - $\mathrm{P}^{4}, \mathrm{M}^{1}$ and $\mathrm{M}^{2}$ are relatively short and broad, the molars have a short emargination; $I_{1}$ is acuspulate; $A_{1}$ and $A_{2}$ are broad; there is a thick bar

Table 1. Measurements of Crocidura kornfeldi teeth from the Somssich Hill 2 locality (for the abbreviations see "Material and methods")

\begin{tabular}{lcccccc}
\hline & & $\mathrm{n}(\mathrm{pcs})$ & $\min .(\mathrm{mm})$ & $\operatorname{mean}(\mathrm{mm})$ & $\max .(\mathrm{mm})$ & $\mathrm{SD}(\mathrm{mm})$ \\
\hline $\mathrm{M}_{1}$ & $\mathrm{~L}$ & 1 & - & 1.61 & - & - \\
& $\mathrm{W}$ & 1 & - & 1.14 & - & - \\
\hline $\mathrm{M}_{2}$ & $\mathrm{~L}$ & 3 & 1.24 & 1.43 & 1.57 & 0.1686 \\
& $\mathrm{~W}$ & 3 & 0.94 & 1.04 & 1.16 & 0.1101 \\
\hline $\mathrm{M}_{3}$ & $\mathrm{~L}$ & 5 & 1.03 & 1.23 & 1.40 & 0.1411 \\
& $\mathrm{~W}$ & 5 & 0.65 & 0.68 & 0.73 & 0.0309 \\
\hline
\end{tabular}




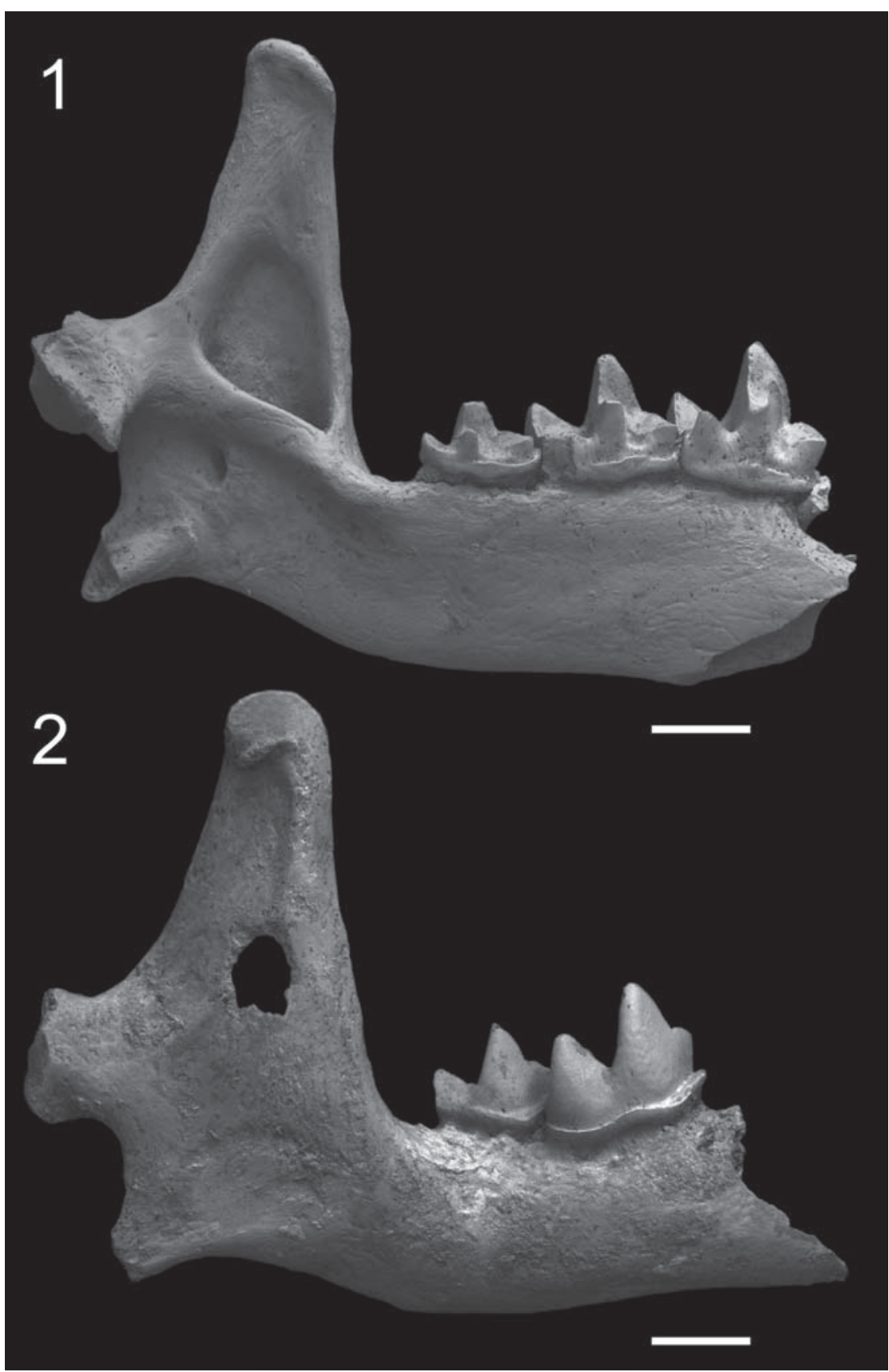

Figs 1-2. Crocidura kornfeldi Kormos, 1934, Somssich Hill 2, scale bar: $1 \mathrm{~mm}$; 1. left mandible fragment with $M_{1}-M_{3}$, layer 30, lingual view; 2. right mandible fragment with $M_{1}-M_{2}$, layer 12 , buccal view 
between the anterior base of the coronoid process and the condyle, underneath the internal temporal fossa; a deep groove is present below this bar (after REUMER 1984).

Emended diagnosis - REUMER (1984) proposed an emended diagnosis, to which Rofes \& CuEnCA-Bescós (2011) added some further observations: $\mathrm{P}^{4}$ and $\mathrm{M}^{1}$ with strong posterior emargination; coronoid spicule either absent or small, poorly developed and situated high, near the tip of the coronoid process; condyle reaching far backwards. We emend the afore-described characters according to RZEBIK-KowALSKA (2000, fig. 13A) and our observations on the Somssich Hill 2 material with the coronoid spicule being indistinct.

Anatomicaldescription - Some detailed anatomical descriptions on C. kornfeldi were already published by several authors (REUMER 1984, RZEBIK-KowALSKA 2000, Koufos et al. 2001, Rofes \& CuenCA-Bescós 2011) coinciding with our observations.

Dentition - The dental morphology of C. kornfeldi is very similar to that of C. obtusa, the detailed description of the teeth is given at C. sp. indet. (kornfeldi or obtusa). In C. kornfeldi, the buccal re-entrant valley on $\mathrm{M}_{1}-\mathrm{M}_{2}$ opens high above the cingulum.

Mandible - The coronoid process is blunt and low. The coronoid spicule is small, situated high and indistinct. The anterior part of the coronoid process leans slightly backwards and the ramus sometimes is broadened at the middle part. The condyle reaches far backwards and is comparatively large in buccal view. The condyle is not high in posterior view, the interarticular area is short. The internal temporal fossa is large and open, reaching to halfway up the coronoid process. The region below the internal temporal fossa (the subfossa) is excavated, separated from the internal temporal fossa by a protruding ridge.

Remarks - Many isolated teeth, fragmented maxillae and mandibles in the material may also belong to this species. However, we determine them as $C$. sp. indet. (kornfeldi or obtusa), because there is no clear evidence for their attachment to C. obtusa.

\section{Crocidura obtusa Kretzoi, 1938}

(Figs 3-4)

1938 Crocidura obtusa n. sp. - KRETzor, p. 92, text-fig. 1a.

1969 Crocidura obtusa Kretzoi - Jánossy, p. 601.

1971 Crocidura obtusa Kretzoi - Koenigswald, p. 120.

2000 Crocidura cf. obtusa Kretzoi - RzeBi K-KowalsKa, pp. 39-43, tabs XXXIV-XXXV, figs 13B, 14.

Holotype - The holotype described by KRETzOI (1938) was one right mandible but it was lost from the collection of the Hungarian Natural History Museum (it was probably destroyed in a devastating fire in 1956) (PÁlfy et al. 2008, p. 140). The original inventory number was Fa. 16. 
Type locality - Gombasek (Gombaszög), Slovakia, Early Pleistocene.

Studied material and measurements - For the overview of the measurements see Table 2. The measurement data of the following list are given in $\mathrm{mm}$.

\section{Layer 4 (VER 2015.255.)}

2.4/4 - Left mandible fragment with $\mathrm{M}_{2} ; \mathrm{L}=1.687, \mathrm{~W}=1.026$

2.4/6 - Left mandible fragment with $\mathrm{M}_{2} ; \mathrm{L}=1.212, \mathrm{~W}=1.080$

2.4/103 - Right mandible fragment

\section{Layer 5 (VER 2015.256.)}

2.5/3 - Left mandible fragment with $\mathrm{M}_{1}$ and $\mathrm{M}_{2} ; \mathrm{M}_{1}: \mathrm{L}=1.728, \mathrm{~W}=1.296 ; \mathrm{M}_{2}: \mathrm{L}=1.512, \mathrm{~W}=1.120$

$2.5 / 13$ - Left mandible fragment with $\mathrm{M}_{3} ; \mathrm{L}=1.269, \mathrm{~W}=0.756$

2.5/53 - Left mandible fragment

\section{Layer 12 (VER 2015.257.)}

2.12/1 - Left mandible fragment with $I_{1}$ fragment, $A_{2}, M_{1}, M_{2}$ and $M_{3} ; M_{1}: L=1.444, W=1.139 ; M_{2}$ : $\mathrm{L}=1.512, \mathrm{~W}=0.972 ; \mathrm{M}_{3}: \mathrm{L}=1.134, \mathrm{~W}=0.675$

\section{Layer 13 (VER 2015.258.)}

2.13/4 - Left mandible fragment

\section{Layer 25 (VER 2015.259.)}

2.25/1 - Left mandible fragment with $A_{1}, M_{1}$ and $M_{2} ; M_{1}: L=1.363, W=0.985 ; M_{2}: L=1.220$, $\mathrm{W}=0.904$

2.25/4 - Left mandible fragment with $\mathrm{M}_{2} ; \mathrm{L}=1.447, \mathrm{~W}=0.877$

\section{Layer 30 (VER 2015.260.)}

2.30/1 - Right mandible fragment

\section{Layer 31 (VER 2015.261.)}

$2.31 / 1$ - Left mandible fragment with eroded $\mathrm{M}_{2}$

Table 2. Measurements of Crocidura obtusa teeth from the Somssich Hill 2 locality (for the abbreviations see "Material and methods")

\begin{tabular}{lcccccc}
\hline & & $\mathrm{n}(\mathrm{pcs})$ & $\mathrm{min} .(\mathrm{mm})$ & mean $(\mathrm{mm})$ & max. $(\mathrm{mm})$ & $\mathrm{SD}(\mathrm{mm})$ \\
\hline $\mathrm{M}_{1}$ & $\mathrm{~L}$ & 3 & 1.36 & 1.51 & 1.73 & 0.1947 \\
& $\mathrm{~W}$ & 3 & 0.99 & 1.14 & 1.30 & 0.1555 \\
\hline $\mathrm{M}_{2}$ & $\mathrm{~L}$ & 6 & 1.21 & 1.43 & 1.69 & 0.1851 \\
& $\mathrm{~W}$ & 6 & 0.88 & 1.00 & 1.12 & 0.0965 \\
\hline $\mathrm{M}_{3}$ & $\mathrm{~L}$ & 2 & 1.13 & 1.20 & 1.27 & 0.0995 \\
& $\mathrm{~W}$ & 2 & 0.68 & 0.72 & 0.76 & 0.0573 \\
\hline
\end{tabular}




\section{Layer 39 (VER 2015.262.)}

2.39/1 - Left mandible fragment

\section{Layer 41 (VER 2015.263.)}

2.41/1 - Left mandible fragment with eroded $\mathrm{M}_{2}$ and $\mathrm{M}_{3}$

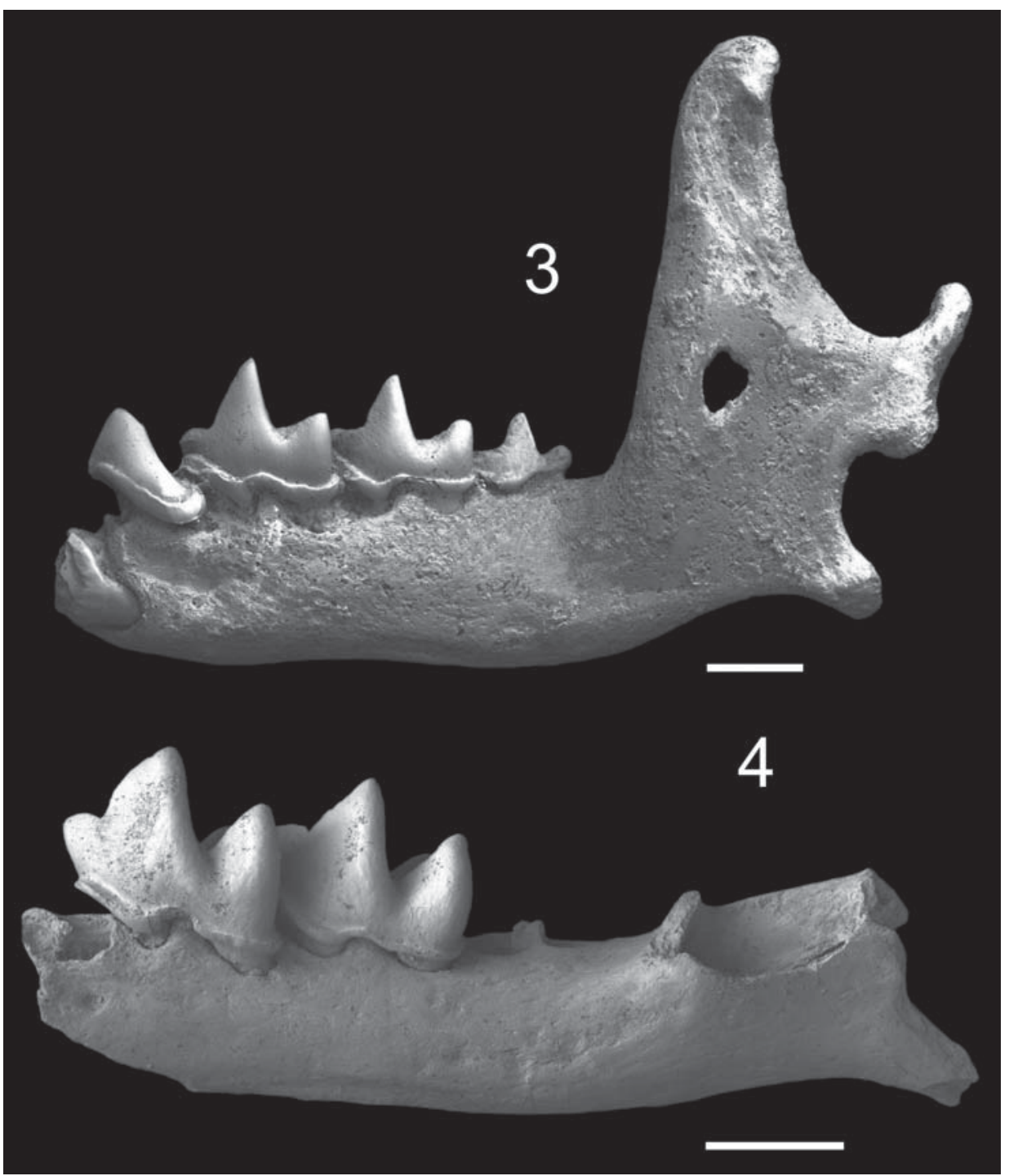

Figs 3-4. Crocidura obtusa Kretzoi, 1938, Somssich Hill 2, scale bar: $1 \mathrm{~mm}$; 3. left mandible with $I_{1}$ fragment, $A_{2}$ and $M_{1}-M_{3}$, layer 12 , buccal view; 4. left mandible fragment with $M_{1}-M_{2}$, layer 5 , buccal view 


\section{Layer 46 (VER 2015.264.)}

2.46/1 - Left mandible fragment

Original diagnosis - KRETzor (1938) distinguished the species from the other similar forms on the basis of the morphology and position of the lower incisor and antemolars.

Emended diagnosis - We emend this diagnosis according to RzEBIKKowALs KA (2000, fig. 13B) and our observations on the Somssich Hill 2 material with the distinct coronoid spicule.

Anatomical description - Up to now mainly short presentations but no detailed descriptions have been born on C. obtusa (KRETZOI 1938, JánOsSY 1969, Koenigswald 1971, RzeBiK-Kowals Ka 2000).

Dentition - The morphological characters of the teeth are very similar to those of $C$. kornfeldi. They are only different in the position of the re-entrant valley of the first and the second lower molars. It opens closer to the buccal cingulum in C. obtusa than in C. kornfeldi.

Mandible - The coronoid process is high, its tip leans strongly backwards. The coronoid spicule is situated high and distinct. The upper part of the condyle reaches far backwards in buccal view. The condyle is high in posterior view, the interarticular area is long. The internal temporal fossa is large and open, reaching to halfway up the coronoid process. The subfossa is sometimes present.

Remarks - Some isolated teeth, maxillary and mandible fragments could be determined as $C$. sp. indet. (kornfeldi or obtusa) (see at C. kornfeldi).

\section{Crocidura sp. indet. (kornfeldi or obtusa)}

(Figs 5-9)

Studied material and measurements - For the overview of the measurements see Table 3. The measurement data of the following list are given in $\mathrm{mm}$.

\section{Layer 4 (VER 2015.305.)}

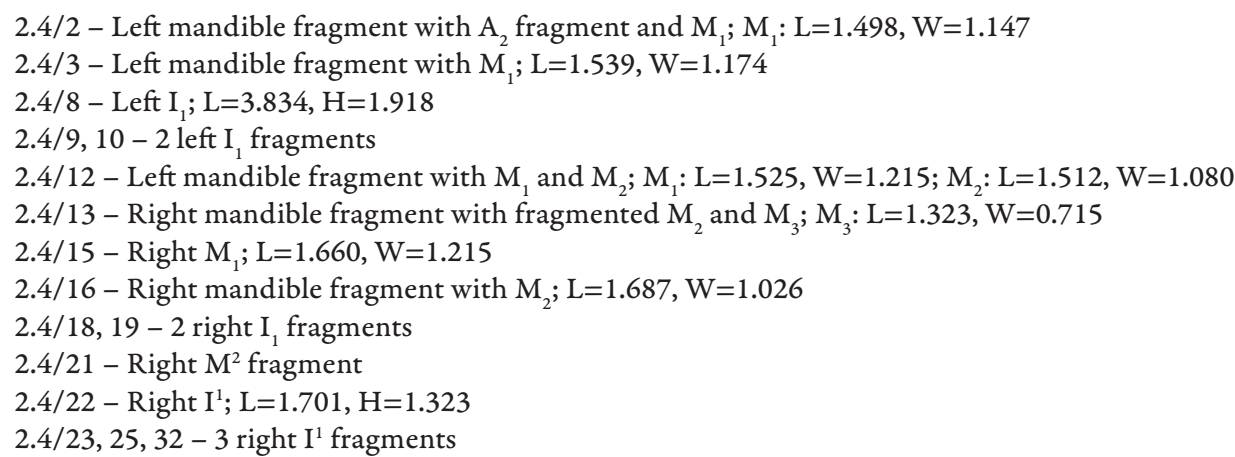


2.4/24 - Right $\mathrm{I}^{1} ; \mathrm{L}=2.160, \mathrm{H}=1.458$

2.4/28 - Left I ${ }^{1}$ fragment

$2.4 / 30$ - Right mandible fragment with $\mathrm{M}_{1} ; \mathrm{L}=1.647, \mathrm{~W}=1.228$

2.4/33 - Left I fragment

2.4/37 - Left mandible fragment

$2.4 / 39$ - Left $\mathrm{M}^{1} ; \mathrm{LL}=1.593, \mathrm{BL}=1.579, \mathrm{AW}=1.755, \mathrm{PW}=2.214$

2.4/40 - Left mandible fragment with $\mathrm{M}_{2} ; \mathrm{L}=1.485, \mathrm{~W}=0.985$

2.4/41, $42-$ Left $\mathrm{A}^{1}$ and $\mathrm{A}^{2}$

2.4/101 - Left mandible fragment with $M_{1}, M_{2}$ and $M_{3} ; M_{1}: L=1.593, W=1.256 ; M_{2}: L=1.350$, $\mathrm{W}=1.066 ; \mathrm{M}_{3}: \mathrm{L}=1.242, \mathrm{~W}=0.675$

$2.4 / 102-$ Left mandible fragment with $\mathrm{M}_{3} ; \mathrm{L}=1.255, \mathrm{~W}=0.715$

\section{Layer 5 (VER 2015.306.)}

2.5/1 - Right mandible fragment with eroded $M_{1}, M_{2}$ and $M_{3}$

$2.5 / 2$ - Right mandible fragment with eroded $A_{1}, M_{1}$ and $M_{2}$

Table 3. Measurements of Crocidura sp. indet. (kornfeldi or obtusa) teeth from the Somssich Hill 2 locality (for the abbreviations see "Material and methods")

\begin{tabular}{lcccccc}
\hline & & $\mathrm{n}(\mathrm{pcs})$ & min. $(\mathrm{mm})$ & mean $(\mathrm{mm})$ & max. $(\mathrm{mm})$ & $\mathrm{SD}(\mathrm{mm})$ \\
\hline $\mathrm{I}^{1}$ & $\mathrm{~L}$ & 17 & 1.66 & 1.97 & 2.16 & 0.1634 \\
& $\mathrm{H}$ & 17 & 1.15 & 1.37 & 1.50 & 0.0895 \\
\hline $\mathrm{P}^{4}$ & $\mathrm{LL}$ & 10 & 0.84 & 1.02 & 1.24 & 0.1284 \\
& $\mathrm{BL}$ & 10 & 1.78 & 1.94 & 2.13 & 0.1045 \\
& $\mathrm{~W}$ & 10 & 1.65 & 1.93 & 2.13 & 0.1441 \\
\hline $\mathrm{M}^{1}$ & $\mathrm{LL}$ & 13 & 1.32 & 1.54 & 1.70 & 0.1066 \\
& $\mathrm{BL}$ & 13 & 1.43 & 1.61 & 1.88 & 0.1384 \\
& $\mathrm{AW}$ & 13 & 1.73 & 1.85 & 1.97 & 0.0618 \\
& $\mathrm{PW}$ & 13 & 2.00 & 2.24 & 2.57 & 0.1444 \\
\hline $\mathrm{M}^{2}$ & $\mathrm{LL}$ & 10 & 1.19 & 1.39 & 1.70 & 0.1440 \\
& $\mathrm{BL}$ & 10 & 1.30 & 1.39 & 1.49 & 0.0605 \\
& $\mathrm{AW}$ & 10 & 2.03 & 2.09 & 2.20 & 0.0720 \\
& $\mathrm{PW}$ & 10 & 1.65 & 1.75 & 1.81 & 0.0481 \\
\hline $\mathrm{I}_{1}$ & $\mathrm{~L}$ & 6 & 3.32 & 3.51 & 3.83 & 0.1798 \\
& $\mathrm{H}$ & 6 & 0.76 & 1.06 & 1.92 & 0.4309 \\
\hline $\mathrm{M}_{1}$ & $\mathrm{~L}$ & 57 & 1.32 & 1.56 & 1.72 & 0.0934 \\
& $\mathrm{~W}$ & 57 & 0.93 & 1.17 & 1.30 & 0.0771 \\
\hline $\mathrm{M}_{2}$ & $\mathrm{~L}$ & 64 & 1.09 & 1.46 & 1.69 & 0.1277 \\
& $\mathrm{~W}$ & 64 & 0.63 & 1.00 & 1.18 & 0.0985 \\
\hline $\mathrm{M}_{3}$ & $\mathrm{~L}$ & 33 & 1.03 & 1.20 & 1.32 & 0.0778 \\
& $\mathrm{~W}$ & 33 & 0.59 & 0.67 & 0.78 & 0.0477 \\
\hline & & & & & &
\end{tabular}


2.5/4 - Left mandible fragment with $\mathrm{M}_{1} ; \mathrm{L}=1.566, \mathrm{~W}=1.215$

$2.5 / 5$ - Right mandible fragment with $\mathrm{M}_{3} ; \mathrm{L}=1.134, \mathrm{~W}=0.621$

2.5/6 - Right mandible fragment with $I_{1}$ and $A_{2}$ fragments and $M_{1} ; M_{1}: L=1.620, W=1.174$

2.5/7 - Left mandible fragment with $\mathrm{M}_{2}$ and $\mathrm{M}_{3} ; \mathrm{M}_{2}: \mathrm{L}=1.593, \mathrm{~W}=1.107 ; \mathrm{M}_{3}: \mathrm{L}=1.215, \mathrm{~W}=0.675$

2.5/8 - Right mandible fragment with $\mathrm{M}_{1} ; \mathrm{L}=1.463, \mathrm{~W}=1.215$

2.5/9 - Left mandible fragment with $I_{1}, A_{1}, A_{2}, M_{1}$ and $M_{2}$ fragments

2.5/11 - Right $M_{1}$ fragment

2.5/14 - Right mandible fragment with $\mathrm{M}_{3} ; \mathrm{L}=1.188, \mathrm{~W}=0.702$

2.5/15 - Right mandible fragment with $M_{1}, M_{2}$ and $M_{3} ; M_{1}: L=1.647, W=1.228 ; M_{2}: L=1.498$,

$\mathrm{W}=1.107 ; \mathrm{M}_{3}: \mathrm{L}=1.269, \mathrm{~W}=0.594$

2.5/16 - Left mandible fragment with $\mathrm{M}_{1}$ and $\mathrm{M}_{2} ; \mathrm{M}_{1}: \mathrm{L}=1.498, \mathrm{~W}=1.134 ; \mathrm{M}_{2}: \mathrm{L}=1.431, \mathrm{~W}=0.972$

$2.5 / 17$ - Right mandible fragment with $\mathrm{A}_{2}$ and $\mathrm{M}_{1} ; \mathrm{M}_{1}: \mathrm{L}=1.620, \mathrm{~W}=1.161$

$2.5 / 18$ - Right mandible fragment with $\mathrm{M}_{2}$ and $\mathrm{M}_{3} ; \mathrm{M}_{2}: \mathrm{L}=1.431, \mathrm{~W}=0.877 ; \mathrm{M}_{3}: \mathrm{L}=1.161, \mathrm{~W}=0.621$

$2.5 / 31$ - Left $\mathrm{I}^{1} ; \mathrm{L}=2.052, \mathrm{H}=1.458$

2.5/32 - Right $\mathrm{I}^{1} ; \mathrm{L}=2.079, \mathrm{H}=1.404$

$2.5 / 33$ - Right $\mathrm{I}^{1} ; \mathrm{L}=2.052, \mathrm{H}=1.323$

$2.5 / 34$ - Left $I^{1}$ fragment

$2.5 / 35$ - Right $\mathrm{I}^{1} ; \mathrm{L}=2.106, \mathrm{H}=1.404$

$2.5 / 36-$ Left $^{1} ; \mathrm{L}=2.079, \mathrm{H}=1.431$

2.5/37 - Right $\mathrm{I}^{1} ; \mathrm{L}=2.160, \mathrm{H}=1.431$

2.5/38 - Left $\mathrm{I}^{1} ; \mathrm{L}=1.944, \mathrm{H}=1.323$

2.5/39 - Left mandible fragment with $\mathrm{M}_{2} ; \mathrm{L}=1.566, \mathrm{~W}=1.066$

$2.5 / 40$ - Left mandible fragment with $\mathrm{M}_{1} ; \mathrm{L}=1.620, \mathrm{~W}=1.161$

$2.5 / 41$ - Left mandible fragment with $\mathrm{M}_{3} ; \mathrm{L}=1.201, \mathrm{~W}=0.783$

2.5/42 - Left mandible fragment with $\mathrm{M}_{1} ; \mathrm{L}=1.593, \mathrm{~W}=1.228$

2.5/43 - Left maxillary fragment with $\mathrm{P}^{4}, \mathrm{M}^{1}$ and $\mathrm{M}^{2} ; \mathrm{P}^{4}: \mathrm{LL}=0.918, \mathrm{BL}=1.971, \mathrm{~W}=1.998 ; \mathrm{M}^{1}$ :

$\mathrm{LL}=1.539, \mathrm{BL}=1.674, \mathrm{AW}=1.890, \mathrm{PW}=2.241 ; \mathrm{M}^{2}: \mathrm{LL}=1.296, \mathrm{BL}=1.350, \mathrm{AW}=2.079$,

$\mathrm{PW}=1.620$

2.5/44 - Right maxillary fragment with $\mathrm{P}^{4}, \mathrm{M}^{1}$ and $\mathrm{M}^{2} ; \mathrm{M}^{1}$ : $\mathrm{LL}=1.593, \mathrm{BL}=1.647, \mathrm{AW}=1.849$,

$\mathrm{PW}=2.268 ; \mathrm{M}^{2}: \mathrm{LL}=1.363, \mathrm{BL}=1.444, \mathrm{AW}=2.065, \mathrm{PW}=1.741$

2.5/45 - Left maxillary fragment with $\mathrm{P}^{4}$ and $\mathrm{M}^{1} ; \mathrm{P}^{4}: \mathrm{LL}=1.161, \mathrm{BL}=2.133, \mathrm{~W}=2.133 ; \mathrm{M}^{1}: \mathrm{LL}=1.647$,

$\mathrm{BL}=1.647, \mathrm{AW}=1.836, \mathrm{PW}=2.295$

2.5/46 - Left maxillary fragment with eroded $\mathrm{A}^{3}$ and $\mathrm{P}^{4}$

2.5/47 - Right maxillary fragment with eroded $\mathrm{I}^{1}$ and $\mathrm{A}^{1}$

2.5/48 - Left $\mathrm{M}^{2}$ fragment

$2.5 / 49$ - Right $\mathrm{M}^{2} ; \mathrm{LL}=1.485, \mathrm{BL}=1.485, \mathrm{AW}=2.160, \mathrm{PW}=1.768$

$2.5 / 50$ - Left maxillary fragment with $\mathrm{P}^{4} ; \mathrm{LL}=0.945, \mathrm{BL}=1.890, \mathrm{~W}=1.863$

$2.5 / 51$ - Right $\mathrm{M}^{2} ; \mathrm{LL}=1.323, \mathrm{BL}=1.350, \mathrm{AW}=2.025, \mathrm{PW}=1.647$

2.5/52 - Eroded right $\mathrm{M}^{2}$

2.5/56 - Right mandible fragment with $\mathrm{M}_{1}$ and $\mathrm{M}_{2} ; \mathrm{M}_{1}: \mathrm{L}=1.404, \mathrm{~W}=1.188 ; \mathrm{M}_{2}: \mathrm{L}=1.431, \mathrm{~W}=1.053$

2.5/61 - Left mandible fragment with $M_{1}, M_{2}$ and $M_{3} ; M_{1}: L=1.431, W=1.269 ; M_{2}: L=1.431$, $\mathrm{W}=1.053 ; \mathrm{M}_{3}: \mathrm{L}=1.188, \mathrm{~W}=0.756$

2.5/62 - Right mandible fragment with $M_{1}, M_{2}$ and $M_{3} ; M_{1}: L=1.485, W=1.161 ; M_{2}: L=1.431$,

$\mathrm{W}=1.080 ; \mathrm{M}_{3}: \mathrm{L}=1.215, \mathrm{~W}=0.729$

2.5/63 - Right mandible fragment with $\mathrm{M}_{2}$ and $\mathrm{M}_{3} ; \mathrm{M}_{2}: \mathrm{L}=1.404, \mathrm{~W}=1.053 ; \mathrm{M}_{3}: \mathrm{L}=1.134, \mathrm{~W}=0.729$

2.5/64 - Right mandible fragment with $M_{1}, M_{2}$ and fragment of $M_{3} ; M_{1}: L=1.620, W=1.188 ; M_{2}$ :

$\mathrm{L}=1.539, \mathrm{~W}=1.053$

2.5/65 - Right mandible fragment with $\mathrm{M}_{1} ; \mathrm{L}=1.647, \mathrm{~W}=1.188$

2.5/66 - Left mandible fragment with $\mathrm{A}_{1}$ and $\mathrm{A}_{2}$ 
2.5/67 - Left mandible fragment with $\mathrm{M}_{2}$ and $\mathrm{M}_{3} ; \mathrm{M}_{2}: \mathrm{L}=1.458, \mathrm{~W}=1.026 ; \mathrm{M}_{3}: \mathrm{L}=1.188, \mathrm{~W}=0.675$

2.5/68 - Left mandible fragment with $M_{1}$ fragment, $M_{2}$ and $M_{3} ; M_{2}: L=1.404, W=1.039 ; M_{3}$ : $\mathrm{L}=1.282, \mathrm{~W}=0.702$

2.5/69 - Left mandible fragment with $\mathrm{M}_{1}$ and $\mathrm{M}_{2} ; \mathrm{M}_{1}: \mathrm{L}=1.485, \mathrm{~W}=1.242 ; \mathrm{M}_{2}: \mathrm{L}=1.390, \mathrm{~W}=1.026$

2.5/72 - Right mandible fragment with $\mathrm{M}_{1}$ and $\mathrm{M}_{2} ; \mathrm{M}_{1}: \mathrm{L}=1.714, \mathrm{~W}=1.269 ; \mathrm{M}_{2}: \mathrm{L}=1.593, \mathrm{~W}=1.161$

2.5/73 - Left mandible fragment with $M_{1}, M_{2}$ and $M_{3} ; M_{1}: L=1.633, W=1.161 ; M_{2}: L=1.458$, $\mathrm{W}=1.053 ; \mathrm{M}_{3}: \mathrm{L}=1.296, \mathrm{~W}=0.729$

2.5/74 - Left mandible fragment with $A_{2}, M_{1}$ and $M_{2} ; M_{1}: L=1.687, W=1.188 ; M_{2}: L=1.566$, $\mathrm{W}=1.053$

\section{Layer 6 (VER 2015.307.)}

2.6/1 - Right mandible fragment with $A_{2} M_{1}$ and $M_{2} ; M_{1}: L=1.647, W=1.155 ; M_{2}: L=1.390$, $\mathrm{W}=0.985$

2.6/2 - Left mandible fragment with $\mathrm{M}_{2}$ and $\mathrm{M}_{3} ; \mathrm{M}_{2}: \mathrm{L}=1.571, \mathrm{~W}=1.066 ; \mathrm{M}_{3}: \mathrm{L}=1.242, \mathrm{~W}=0.742$

2.6/3 - Right mandible fragment with $A_{2}, M_{1}$ and $M_{2} ; M_{1}: L=1.606, W=1.120 ; M_{2}: L=1.436$, $\mathrm{W}=1.031$

$2.6 / 5$ - Left mandible fragment with $\mathrm{M}_{2} ; \mathrm{L}=1.323, \mathrm{~W}=0.896$

2.6/6 - Right mandible fragment with $\mathrm{M}_{2}$ and $\mathrm{M}_{3} ; \mathrm{M}_{2}: \mathrm{L}=1.487, \mathrm{~W}=1.053 ; \mathrm{M}_{3}: \mathrm{L}=1.293, \mathrm{~W}=0.675$

$2.6 / 7-$ Left $\mathrm{I}_{1} ; \mathrm{L}=3.391, \mathrm{H}=0.866$

2.6/8 - Left I fragment

2.6/9 - Left maxillary fragment with $\mathrm{P}^{4}$ and $\mathrm{M}^{1} ; \mathrm{P}^{4}: \mathrm{LL}=1.080, \mathrm{BL}=2.052, \mathrm{~W}=2.049 ; \mathrm{M}^{1}: \mathrm{LL}=1.647$, $\mathrm{BL}=1.679, \mathrm{AW}=1.890, \mathrm{PW}=2.362$

2.6/10 - Left $\mathrm{M}^{2} ; \mathrm{LL}=1.379, \mathrm{BL}=1.409, \mathrm{AW}=2.203, \mathrm{PW}=1.795$

2.6/11 - Left $\mathrm{I}^{1} ; \mathrm{L}=2.052, \mathrm{H}=1.428$

2.6/12 - Eroded left I ${ }^{1}$

2.6/13 - Left $\mathrm{I}^{1} ; \mathrm{L}=2.146, \mathrm{H}=1.385$

\section{Layer 7 (VER 2015.308.)}

2.7/1 - Right mandible fragment with eroded $\mathrm{M}_{2}$ and $\mathrm{M}_{3}$

$2.7 / 3$ - Right $\mathrm{I}^{1} ; \mathrm{L}=1.922, \mathrm{H}=1.339$

2.7/4 - Fragmented right $\mathrm{M}_{3}$

\section{Layer 8 (VER 2015.309.)}

2.8/1 - Left mandible fragment with $\mathrm{M}_{1}$ and $\mathrm{M}_{2} ; \mathrm{M}_{1}: \mathrm{L}=1.606, \mathrm{~W}=1.215 ; \mathrm{M}_{2}: \mathrm{L}=1.539, \mathrm{~W}=1.053$

$2.8 / 2$ - Left mandible with complete dentition; $\mathrm{I}_{1}: \mathrm{L}=3.685, \mathrm{H}=1.026 ; \mathrm{M}_{1}: \mathrm{L}=1.728, \mathrm{~W}=1.242 ; \mathrm{M}_{2}$ : $\mathrm{L}=1.512, \mathrm{~W}=0.999 ; \mathrm{M}_{3}: \mathrm{L}=1.296, \mathrm{~W}=0.680$

2.8/5 - Right mandible fragment with $A_{2}, M_{1}, M_{2}$ and $M_{3} ; M_{1}: L=1.377, W=1.215 ; M_{2}: L=1.571$, $\mathrm{W}=1.080 ; \mathrm{M}_{3}: \mathrm{L}=1.255, \mathrm{~W}=0.677$

2.8/6 - Left maxillary fragment with $\mathrm{A}^{2}, \mathrm{~A}^{3}, \mathrm{P}^{4}, \mathrm{M}^{1}$ and $\mathrm{M}^{2} ; \mathrm{P}^{4}: \mathrm{LL}=0.999, \mathrm{BL}=1.922, \mathrm{~W}=1.957$; $\mathrm{M}^{1}: \mathrm{LL}=1.458, \mathrm{BL}=1.471, \mathrm{AW}=1.890, \mathrm{PW}=2.025 ; \mathrm{M}^{2}: \mathrm{LL}=1.474, \mathrm{BL}=1.330, \mathrm{AW}=2.052$, $\mathrm{PW}=1.728$

2.8/11 - Left mandible fragment with $M_{1}, M_{2}$ and $M_{3}$ fragment; $M_{1}: L=1.660, W=1.239 ; M_{2}$ : $\mathrm{L}=1.485, \mathrm{~W}=1.082$

2.8/12 - Right $\mathrm{I}^{1}$ fragment

2.8/13 - Right $\mathrm{M}^{1} ; \mathrm{LL}=1.525, \mathrm{BL}=1.498, \mathrm{AW}=1.860, \mathrm{PW}=2.268$

2.8/14 - Left I fragment 


\section{Layer 9 (VER 2015.310.)}

2.9/1 - Left mandible fragment with $\mathrm{M}_{2}$ and $\mathrm{M}_{3} ; \mathrm{M}_{2}: \mathrm{L}=1.517, \mathrm{~W}=0.945 ; \mathrm{M}_{3}: \mathrm{L}=1.212, \mathrm{~W}=0.672$

2.9/2 - Right mandible fragment with $\mathrm{I}_{1}, \mathrm{~A}_{1}, \mathrm{~A}_{2}$ fragment and $\mathrm{M}_{1} ; \mathrm{M}_{1}: \mathrm{L}=1.498, \mathrm{~W}=1.066$

$2.9 / 3$ - Left mandible fragment with $\mathrm{I}_{1}, \mathrm{~A}_{1}$ and $\mathrm{A}_{2} ; \mathrm{I}_{1}: \mathrm{L}=3.469, \mathrm{H}=0.891$

2.9/4 - Left $\mathrm{I}^{1} ; \mathrm{L}=1.660, \mathrm{H}=1.147$

\section{Layer 10 (VER 2015.311.)}

2.10/11 - Left mandible fragment with $\mathrm{A}_{2}, \mathrm{M}_{1}, \mathrm{M}_{2}$ and $\mathrm{M}_{3} ; \mathrm{M}_{1}: \mathrm{L}=1.474, \mathrm{~W}=1.163 ; \mathrm{M}_{2}: \mathrm{L}=1.201$, $\mathrm{W}=0.904 ; \mathrm{M}_{3}: \mathrm{L}=1.188, \mathrm{~W}=0.704$

2.10/12 - Left mandible fragment with $\mathrm{M}_{1}, \mathrm{M}_{2}$ and $\mathrm{M}_{3} ; \mathrm{M}_{1}: \mathrm{L}=1.674, \mathrm{~W}=1.212 ; \mathrm{M}_{2}: \mathrm{L}=1.597$, $\mathrm{W}=1.088 ; \mathrm{M}_{3}: \mathrm{L}=1.269, \mathrm{~W}=0.702$

2.10/13 - Right mandible fragment with $M_{1}$ fragment, $M_{2}$ and $M_{3} ; M_{2}: L=1.437, W=1.026 ; M_{3}$ : $\mathrm{L}=1.026, \mathrm{~W}=0.599$

2.10/14 - Left mandible fragment with $\mathrm{M}_{1}$ and $\mathrm{M}_{2} ; \mathrm{M}_{1}: \mathrm{L}=1.544, \mathrm{~W}=1.120 ; \mathrm{M}_{2}: \mathrm{L}=1.296, \mathrm{~W}=0.972$

2.10/15 - Right mandible fragment with $\mathrm{M}_{1}$ and $\mathrm{M}_{3} ; \mathrm{M}_{1}: \mathrm{L}=1.722, \mathrm{~W}=1.085 ; \mathrm{M}_{3}: \mathrm{L}=1.190$, $\mathrm{W}=0.648$

2.10/16 - Right mandible fragment with $\mathrm{I}_{1}, \mathrm{~A}_{1}$ and $\mathrm{A}_{2} ; \mathrm{I}_{1}: \mathrm{L}=3.577, \mathrm{H}=1.053$

2.10/17 - Right mandible fragment with $\mathrm{M}_{1}$ and $\mathrm{M}_{2} ; \mathrm{M}_{1}: \mathrm{L}=1.674, \mathrm{~W}=1.212 ; \mathrm{M}_{2}: \mathrm{L}=1.490$, $\mathrm{W}=1.080$

2.10/18 - Right mandible fragment with $\mathrm{M}_{2}$ and $\mathrm{M}_{3}$ fragment; $\mathrm{M}_{2}: \mathrm{L}=1.593, \mathrm{~W}=1.177$

2.10/19 - Right mandible fragment with $M_{1}$ and $M_{2} ; M_{1}: L=1.409, W=1.188 ; M_{2}: L=1.471$, $\mathrm{W}=1.080$

2.10/20 - Right mandible fragment with $\mathrm{M}_{2} ; \mathrm{L}=1.552, \mathrm{~W}=1.080$

2.10/21 - Left maxillary fragment with $\mathrm{P}^{4}$ and $\mathrm{M}^{1}$ fragment and $\mathrm{M}^{2} ; \mathrm{M}^{2}: \mathrm{LL}=1.296, \mathrm{BL}=1.444$, $\mathrm{AW}=2.030, \mathrm{PW}=1.757$

2.10/22 - Left maxillary fragment with $\mathrm{P}^{4}$ and $\mathrm{M}^{1} ; \mathrm{P}^{4}: \mathrm{LL}=0.837, \mathrm{BL}=1.895, \mathrm{~W}=1.647 ; \mathrm{M}^{1}$ : $\mathrm{LL}=1.458, \mathrm{BL}=1.876, \mathrm{AW}=1.906, \mathrm{PW}=2.173$

2.10/23 - Left maxillary fragment with $\mathrm{M}^{1}$ and $\mathrm{M}^{2} ; \mathrm{M}^{1}: \mathrm{LL}=1.701, \mathrm{BL}=1.512, \mathrm{AW}=1.944$, $\mathrm{PW}=2.322 ; \mathrm{M}^{2}: \mathrm{LL}=1.377, \mathrm{BL}=1.458, \mathrm{AW}=2.187, \mathrm{PW}=1.757$

2.10/24 - Right $\mathrm{I}^{1}$ fragment

\section{Layer 11 (VER 2015.312.)}

2.11/1 - Right mandible fragment with $\mathrm{M}_{1}, \mathrm{M}_{2}$ and $\mathrm{M}_{3} ; \mathrm{M}_{1}: \mathrm{L}=1.512, \mathrm{~W}=1.209 ; \mathrm{M}_{2}: \mathrm{L}=1.539$, $\mathrm{W}=1.004 ; \mathrm{M}_{3}: \mathrm{L}=1.131, \mathrm{~W}=0.645$

2.11/2 - Left mandible fragment with $\mathrm{A}_{2}, \mathrm{M}_{1}$ and $\mathrm{M}_{2}$ fragment; $\mathrm{M}_{1}: \mathrm{L}=1.593, \mathrm{~W}=1.215$

2.11/3 - Left mandible fragment with $M_{1}, M_{2}$ and $M_{3}$ fragments

2.11/4 - Right mandible fragment with $\mathrm{M}_{1}$ and $\mathrm{M}_{2} ; \mathrm{M}_{1}: \mathrm{L}=1.620, \mathrm{~W}=1.236 ; \mathrm{M}_{2}: \mathrm{L}=1.485, \mathrm{~W}=1.080$

$2.11 / 5$ - Left mandible fragment with $A_{2}$ and $M_{1}, M_{2}$ fragments

$2.11 / 6$ - Right $\mathrm{I}^{1}$ fragment

\section{Layer 12 (VER 2015.313.)}

$2.12 / 3$ - Right mandible fragment with $A_{1}, A_{2}, M_{1}$ and $M_{2} ; M_{1}: L=1.660, W=1.215 ; M_{2}: L=1.660$, $\mathrm{W}=0.945$

2.12/4 - Skull fragment with left $\mathrm{I}^{1}$ fragment, $\mathrm{A}^{1}, \mathrm{~A}^{2}, \mathrm{~A}^{3}, \mathrm{P}^{4}$ fragment, right $\mathrm{A}^{2}, \mathrm{~A}^{3}, \mathrm{P}^{4}$ and $\mathrm{M}^{1}$ fragment; $\mathrm{P}^{4}: \mathrm{LL}=1.053, \mathrm{BL}=2.030, \mathrm{~W}=1.998$

2.12/6 - Right mandible fragment with $\mathrm{M}_{3} ; \mathrm{L}=1.215, \mathrm{~W}=0.675$ 
2.12/7 - Left mandible fragment with $\mathrm{M}_{2}$ and $\mathrm{M}_{3} ; \mathrm{M}_{2}: \mathrm{L}=1.566, \mathrm{~W}=0.985 ; \mathrm{M}_{3}: \mathrm{L}=1.188, \mathrm{~W}=0.675$

2.12/8 - Left mandible fragment with $\mathrm{M}_{2}$ and $\mathrm{M}_{3}$ fragments

2.12/9 - Right I fragment

$2.12 / 10-$ Right $I_{1} ; \mathrm{L}=3.321, \mathrm{H}=0.756$

\section{Layer 13 (VER 2015.314.)}

2.13/2 - Left maxillary fragment with $\mathrm{P}^{4}, \mathrm{M}^{1}$ and $\mathrm{M}^{2} ; \mathrm{P}^{4}: \mathrm{LL}=0.864, \mathrm{BL}=1.849, \mathrm{~W}=1.755 ; \mathrm{M}^{1}$ : $\mathrm{LL}=1.431, \mathrm{BL}=1.485, \mathrm{AW}=1.728, \mathrm{PW}=2.165 ; \mathrm{M}^{2}: \mathrm{LL}=1.185, \mathrm{BL}=1.298, \mathrm{AW}=2.025$, $\mathrm{PW}=1.809$

2.13/3 - Right maxillary fragment with $\mathrm{M}^{1}$ and $\mathrm{M}^{2} ; \mathrm{M}^{1}: \mathrm{LL}=1.593, \mathrm{BL}=1.566, \mathrm{AW}=1.809$, $\mathrm{PW}=2.565 ; \mathrm{M}^{2}: \mathrm{LL}=1.701, \mathrm{BL}=1.377, \mathrm{AW}=2.025, \mathrm{PW}=1.701$

2.13/5 - Left maxillary fragment with $\mathrm{M}^{1}$ and $\mathrm{M}^{2} ; \mathrm{M}^{1}: \mathrm{LL}=1.593, \mathrm{BL}=1.566, \mathrm{AW}=2.106, \mathrm{PW}=2.268$; $\mathrm{M}^{2}: \mathrm{LL}=1.458, \mathrm{BL}=1.390, \mathrm{AW}=2.254, \mathrm{PW}=1.755$

2.13/6 - Right mandible fragment with $\mathrm{M}_{2}$ and $\mathrm{M}_{3} ; \mathrm{M}_{2}: \mathrm{L}=1.323, \mathrm{~W}=0.864 ; \mathrm{M}_{3}: \mathrm{L}=1.053, \mathrm{~W}=0.594$

2.13/7 - Right mandible fragment with $\mathrm{M}_{2}$ and $\mathrm{M}_{3} ; \mathrm{M}_{2}: \mathrm{L}=1.458, \mathrm{~W}=0.931 ; \mathrm{M}_{3}: \mathrm{L}=1.107, \mathrm{~W}=0.621$

2.13/8 - Eroded right $\mathrm{M}_{2}$

$2.13 / 9$ - Eroded right $\mathrm{I}^{1}$

\section{Layer 14(VER 2015.315.)}

2.14/2 - Right mandible fragment with $\mathrm{M}_{1}$ and $\mathrm{M}_{2} ; \mathrm{M}_{1}: \mathrm{L}=1.323, \mathrm{~W}=0.945 ; \mathrm{M}_{2}: \mathrm{L}=1.444, \mathrm{~W}=0.810$

$2.14 / 3$ - Right mandible fragment with $\mathrm{M}_{2}$ and $\mathrm{M}_{3}$ fragment; $\mathrm{M}_{2}: \mathrm{L}=1.593, \mathrm{~W}=1.026$

2.14/4 - Right mandible fragment with $\mathrm{A}_{2}$ fragment, eroded $\mathrm{M}_{1}$ and $\mathrm{M}_{2}$

$2.14 / 5$ - Left mandible fragment with $\mathrm{A}_{1}, \mathrm{~A}_{2}$ and $\mathrm{M}_{1} ; \mathrm{M}_{1}: \mathrm{L}=1.582, \mathrm{~W}=1.201$

\section{Layer 15 (VER 2015.316.)}

2.15/1 - Right maxillary fragment with $\mathrm{M}^{1}$ and $\mathrm{M}^{2} ; \mathrm{M}^{1}: \mathrm{LL}=1.539, \mathrm{BL}=1.620, \mathrm{AW}=1.849$, $\mathrm{PW}=2.214 ; \mathrm{M}^{2}: \mathrm{LL}=1.323, \mathrm{BL}=1.431, \mathrm{AW}=2.133, \mathrm{PW}=1.782$

\section{Layer 16 (VER 2015.317.)}

$2.16 / 1$ - Left mandible fragment with $M_{1} ; M_{1}: L=1.647, W=1.174$

$2.16 / 2$ - Right mandible fragment with $\mathrm{I}_{1}$ fragment, $\mathrm{A}_{1}, \mathrm{~A}_{2}$ and $\mathrm{M}_{1} ; \mathrm{M}_{1}: \mathrm{L}=1.552, \mathrm{~W}=1.174$

$2.16 / 4$ - Right $\mathrm{M}_{1} ; \mathrm{L}=1.579, \mathrm{~W}=1.166$

\section{Layer 18 (VER 2015.318.)}

2.18/1 - Right mandible fragment with $\mathrm{A}_{1}, \mathrm{~A}_{2}, \mathrm{M}_{1}$ and $\mathrm{M}_{2}$ fragment; $\mathrm{M}_{1}: \mathrm{L}=1.566, \mathrm{~W}=1.147$

\section{Layer 20 (VER 2015.319.)}

2.20/1 - Right maxillary fragment with $\mathrm{P}^{4}, \mathrm{M}^{1}$ and $\mathrm{M}^{2}$ fragments

\section{Layer 22 (VER 2015.320.)}

2.22/1 - Left maxillary fragment with $\mathrm{M}^{1}$ and $\mathrm{M}^{2} ; \mathrm{M}^{1}: \mathrm{LL}=1.458, \mathrm{BL}=1.647, \mathrm{AW}=1.863, \mathrm{PW}=2.187$;

$\mathrm{M}^{2}: \mathrm{LL}=1.323, \mathrm{BL}=1.363, \mathrm{AW}=2.106, \mathrm{PW}=1.728$

$2.22 / 2$ - Left mandible fragment with $\mathrm{M}_{3} ; \mathrm{L}=1.058, \mathrm{~W}=0.648$

2.22/11 - Right mandible fragment with $\mathrm{M}_{1}$ fragment and $\mathrm{M}_{2} ; \mathrm{M}_{2}: \mathrm{L}=1.363, \mathrm{~W}=0.904$

2.22/12 - Left mandible fragment with $\mathrm{M}_{3} ; \mathrm{L}=1.080, \mathrm{~W}=0.642$ 


\section{Layer 24 (VER 2015.321.)}

2.24/1 - Right mandible fragment with $\mathrm{M}_{2}$ and $\mathrm{M}_{3} ; \mathrm{M}_{2}: \mathrm{L}=1.336, \mathrm{~W}=0.869 ; \mathrm{M}_{3}: \mathrm{L}=1.093, \mathrm{~W}=0.637$

\section{Layer 25 (VER 2015.322.)}

2.25/2 - Left mandible fragment with $\mathrm{M}_{1}$ and $\mathrm{M}_{2} ; \mathrm{M}_{1}: \mathrm{L}=1.431, \mathrm{~W}=1.080 ; \mathrm{M}_{2}: \mathrm{L}=1.350, \mathrm{~W}=0.891$

2.25/3 - Left mandible fragment with $\mathrm{M}_{1}, \mathrm{M}_{2}$ and $\mathrm{M}_{3} ; \mathrm{M}_{1}: \mathrm{L}=1.620, \mathrm{~W}=1.269 ; \mathrm{M}_{2}: \mathrm{L}=1.512$, $\mathrm{W}=1.080 ; \mathrm{M}_{3}: \mathrm{L}=1.309, \mathrm{~W}=0.642$

\section{Layer 26 (VER 2015.323.)}

$2.26 / 1$ - Eroded right $\mathrm{I}^{1}$

$2.26 / 2$ - Left mandible fragment with $\mathrm{M}_{1}$ and $\mathrm{M}_{2} ; \mathrm{M}_{1}: \mathrm{L}=1.539, \mathrm{~W}=1.188 ; \mathrm{M}_{2}: \mathrm{L}=1.512, \mathrm{~W}=1.053$ $2.26 / 3$ - Right $\mathrm{M}_{2} ; \mathrm{L}=1.587, \mathrm{~W}=1.039$

\section{Layer 29 (VER 2015.324.)}

2.29/1 - Right mandible fragment with eroded $\mathrm{M}_{2}$

$2.29 / 2$ - Right mandible fragment with $\mathrm{M}_{2} ; \mathrm{L}=1.485, \mathrm{~W}=0.972$

$2.29 / 3$ - Left mandible fragment with $\mathrm{A}_{2}$ and $\mathrm{M}_{1} ; \mathrm{M}_{1}: \mathrm{L}=1.566, \mathrm{~W}=1.147$

2.29/4 - Right mandible fragment

\section{Layer 31 (VER 2015.325.)}

2.31/3 - Right mandible fragment with $\mathrm{A}_{1}, \mathrm{~A}_{2}$ and $\mathrm{M}_{1} ; \mathrm{M}_{1}: \mathrm{L}=1.633, \mathrm{~W}=1.188$

2.31/4 - Left mandible fragment with $\mathrm{M}_{1}$ and $\mathrm{M}_{3} ; \mathrm{M}_{1}: \mathrm{L}=1.593, \mathrm{~W}=1.107 ; \mathrm{M}_{3}: \mathrm{L}=1.188, \mathrm{~W}=0.661$

\section{Layer 33 (VER 2015.326.)}

2.33/2 - Right mandible fragment with $\mathrm{M}_{1}, \mathrm{M}_{2}$ and $\mathrm{M}_{3} ; \mathrm{M}_{1}: \mathrm{L}=1.431, \mathrm{~W}=1.058 ; \mathrm{M}_{2}: \mathrm{L}=1.409$, $\mathrm{W}=0.934 ; \mathrm{M}_{3}: \mathrm{L}=1.134, \mathrm{~W}=0.618$

$2.33 / 3-$ Right $^{1} ; \mathrm{L}=1.782, \mathrm{H}=1.498$

\section{Layer 36 (VER 2015.327.)}

2.36/1 - Left maxillary fragment with $\mathrm{P}^{4} ; \mathrm{LL}=1.242, \mathrm{BL}=1.782, \mathrm{~W}=1.998$

\section{Layer 37 (VER 2015.328.)}

2.37/2 - Right mandible fragment with $\mathrm{M}_{1}$ fragment and $\mathrm{M}_{2} ; \mathrm{M}_{2}: \mathrm{L}=1.282, \mathrm{~W}=0.931$

$2.37 / 3$ - Left mandible fragment with $\mathrm{M}_{2}$ and $\mathrm{M}_{3} ; \mathrm{M}_{2}: \mathrm{L}=1.404, \mathrm{~W}=0.877 ; \mathrm{M}_{3}: \mathrm{L}=1.112, \mathrm{~W}=0.634$ $2.37 / 4-$ Left $^{1} ; \mathrm{L}=1.755, \mathrm{H}=1.228$

\section{Layer 38 (VER 2015.329.)}

2.38/1 - Left mandible fragment with $\mathrm{M}_{1}$ and $\mathrm{M}_{2} ; \mathrm{M}_{1}: \mathrm{L}=1.433, \mathrm{~W}=0.985 ; \mathrm{M}_{2}: \mathrm{L}=1.350, \mathrm{~W}=0.918$ $2.38 / 2$ - Left $\mathrm{I}^{1} ; \mathrm{L}=1.876, \mathrm{H}=1.296$

2.38/3 - Left $\mathrm{M}_{2} ; \mathrm{L}=1.552, \mathrm{~W}=1.039$

2.38/4 - Right $\mathrm{I}^{1} ; \mathrm{L}=1.971, \mathrm{H}=1.363$

$2.38 / 5-$ Left $\mathrm{M}^{1} ; \mathrm{LL}=1.323, \mathrm{BL}=1.433, \mathrm{AW}=1.795, \mathrm{PW}=1.998$

$2.38 / 6$ - Right $\mathrm{M}_{1} ; \mathrm{L}=1.444, \mathrm{~W}=1.058$ 


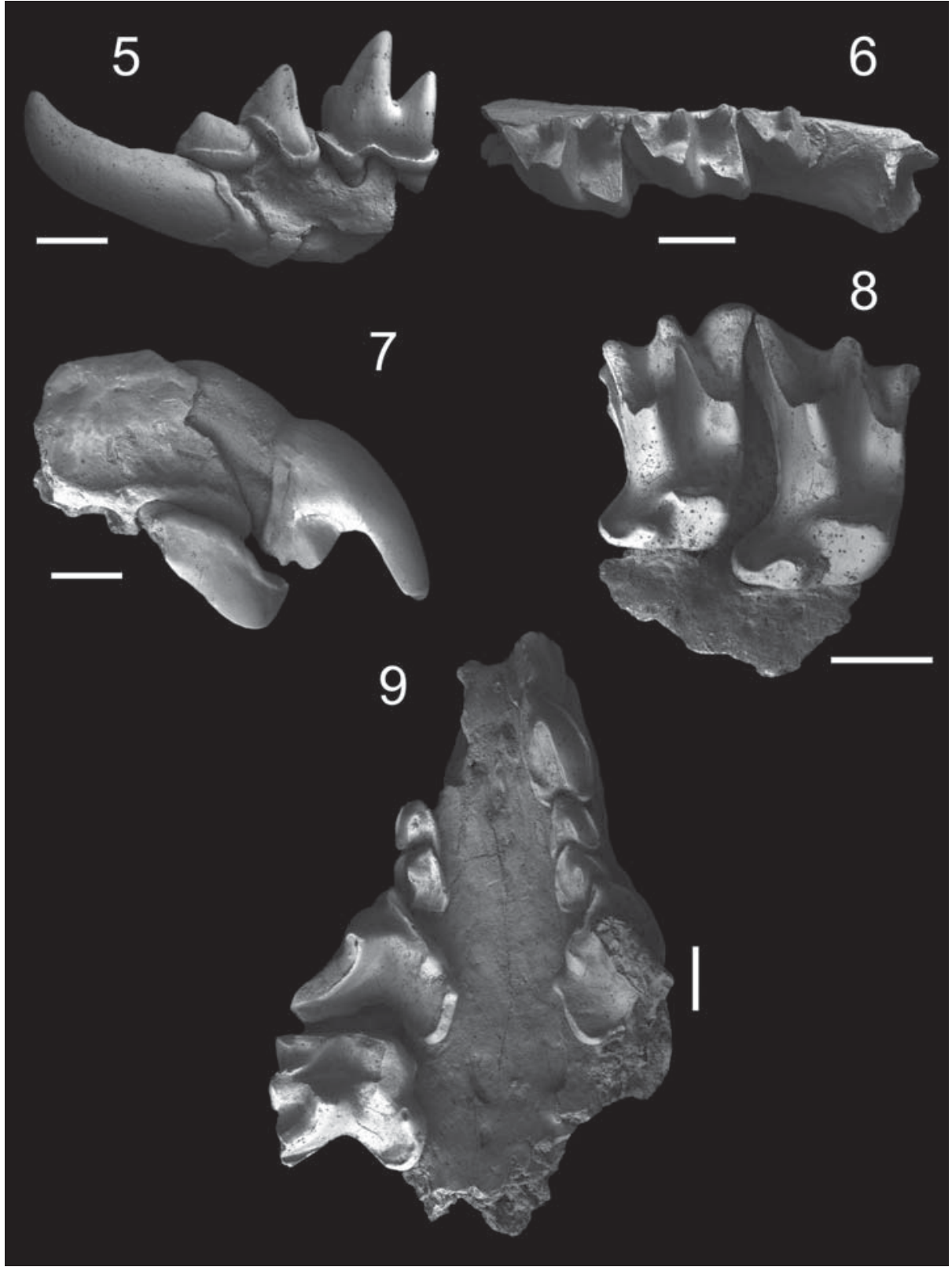

Figs 5-9. Crocidura sp. indet. (kornfeldi or obtusa), Somssich Hill 2, scale bar: $1 \mathrm{~mm}$; 5. left mandible fragment with $I_{1}-M_{1}$, layer 45 , buccal view; 6 . left mandible fragment with $M_{1}-M_{3}$, layer 10 , occlusal view; 7. right maxillary fragment with $\mathrm{I}^{1}-\mathrm{A}^{1}$, layer 5 , buccal view; 8. right maxillary fragment with $\mathrm{M}^{1}-\mathrm{M}^{2}$, layer 5, occlusal view; 9. skull fragment with left $\mathrm{I}^{1}-\mathrm{P}^{4}$ and right $\mathrm{A}^{2}-\mathrm{M}^{1}$, layer 12 , occlusal view 
Layer 41 (VER 2015.330.)

2.41/2 - Left mandible fragment with $\mathrm{M}_{2}$ and $\mathrm{M}_{3}$ fragment; $\mathrm{M}_{2}: \mathrm{L}=1.485, \mathrm{~W}=0.977$

Layer 42 (VER 2015.331.)

2.42/1 - Right mandible fragment with $\mathrm{M}_{2} ; \mathrm{L}=1.350, \mathrm{~W}=1.001$

Layer 43 (VER 2015.332.)

2.43/1 - Left mandible fragment with $\mathrm{M}_{2} ; \mathrm{L}=1.161, \mathrm{~W}=0.931$

Layer 44 (VER 2015.333.)

2.44/1 - Left maxillary fragment with $\mathrm{A}^{1}, \mathrm{~A}^{2}, \mathrm{~A}^{3}$ and $\mathrm{P}^{4}$ fragment

Layer 45 (VER 2015.334.)

2.45/2 - Left mandible fragment with $\mathrm{I}_{1}, \mathrm{~A}_{1}, \mathrm{~A}_{2}$ and $\mathrm{M}_{1} ; \mathrm{I}_{1}: \mathrm{L}=3.483, \mathrm{H}=0.877 ; \mathrm{M}_{1}: \mathrm{L}=1.593$, $\mathrm{W}=1.147$

Layer 46 (VER 2015.335.)

$2.46 / 2$ - Left maxillary fragment with $\mathrm{A}^{2}, \mathrm{~A}^{3}$ and $\mathrm{P}^{4} ; \mathrm{P}^{4}: \mathrm{LL}=1.053, \mathrm{BL}=1.903, \mathrm{~W}=1.890$

Anatomical description - The identification of isolated teeth of the genus Crocidura is very difficult. The majority of the isolated teeth of the early Crocidura species in Europe are referred as Crocidura sp.

Dentition $-I^{1}-$ The apex is pointed and the talon also has a little, sharp cone. The cingulum along the posterior buccal margin is narrow but well-pronounced, usually it is undulate.

$\boldsymbol{A}^{1}-\boldsymbol{A}^{3}$ - The first antemolar is the largest, the two posterior ones are considerably smaller. $\mathrm{A}^{2}$ is significantly smaller than $\mathrm{A}^{3}$. The talon of $\mathrm{A}^{3}$ is hidden under the parastyle of the $\mathrm{P}^{4}$. Cingula are well-developed on both sides of the upper antemolars.

$P^{4}$ - The parastyle of $\mathrm{P}^{4}$ is protruding and separated from the paracone by a deep valley. No parastylar crest is present. The protocone is small and situated buccally to the anterolingual corner, which is therefore rounded. A small hypocone is visible on the cingulum-like ridge running along the lingual margin of the tooth. This hypoconal ridge is separated from the protocone by a valley. The posterior emargination is strong.

$\boldsymbol{M}^{1}-\boldsymbol{M}^{2}$ - Both of the upper molars are relatively broad and short. The protocone is connected to the paracone, but between the protocone and the metacone there is a wide and deep valley. The hypocone is situated posterolingually to the protocone. It is poorly defined and separated from the protocone by a valley. On the $\mathrm{M}^{1}$, the posterobuccal corner protrudes strongly, the metastyle is straight, while in the anterobuccal corner a little, curved parastyle is present. The $\mathrm{M}^{1} \mathrm{AW}$ is far smaller than the PW. The shape of $\mathrm{M}^{2}$ is trapezoidal, its anterior part is far 
wider than the posterior one. The parastyle on $\mathrm{M}^{2}$ is long and curved, while the metastyle is short and straight.

$\boldsymbol{M}^{3}$ - It is not present in the Somssich Hill 2 material.

$I_{1}$ - The apex is upturned, the dorsal margin is slightly bicuspulate. The buccal cingulum is narrow but pronounced. $\mathrm{I}_{1}$ reaches back to the posterior end of $\mathrm{A}_{1}$, underneath $\mathrm{A}_{2}$.

$\boldsymbol{A}_{1}$ - This element is anteroposteriorly quite elongate, only a small part of it is hidden underneath $\mathrm{A}_{2}$. The cingula are well-developed on both sides.

$\boldsymbol{A}_{2}-\mathrm{A}_{2}$ is typical for Crocidura: a high, pointed, tetrahedron-shaped tooth. The cingula are equally strong on both sides.

$\boldsymbol{M}_{1}-\boldsymbol{M}_{2}$ - The lower molars are also typical for Crocidura. The entoconid crest is usually absent. The buccal cingulum is narrow but well-pronounced. It is undulate in all specimens, but it is less undulate on $M_{2}$ than on $M_{1}$. The lingual cingulum is weak.

$\boldsymbol{M}_{3}$ - The talonid of $\mathrm{M}_{3}$ is reduced to a single cuspid, which is the hypoconid. The development of the cingula is as in $M_{1}$ and $M_{2}$.

\section{DISCUSSION}

There are three Crocidura species reported from the Early Pleistocene of the European mainland. Crocidura zorzii Pasa, 1942 (Italy, KotsAKIs et al. 2003) is larger in size than the specimens we found here. The forms reported here can be determined on the basis of morphology and measurements as C. kornfeldi or C. obtusa.

These two Crocidura species can be distinguished morphologically by the characteristics of the mandible. The ramus mandibulae of $C$. kornfeldi is blunter and lower. The tip of the coronoid process of C. obtusa leans strongly backwards. The coronoid spicule of C. obtusa is distinct, while it is indistinct in C. kornfeldi. The condyle is higher and the interarticular area is longer in C. obtusa than in C. kornfeldi.

On the $M_{1}-M_{2}$, the buccal re-entrant valley opens higher above the cingulum in C. kornfeldi, than in C. obtusa, but in the case of the isolated teeth it is not useful for the precise identification.

The isolated teeth may be determined by means of morphometrical methods as well. The $M_{1} L / W$ rate does not show any segregation within the two species (Fig. 10). Nonetheless, we can see some detachments on the scatter plots of the $\mathrm{M}_{2}$ and $\mathrm{M}_{3} \mathrm{~L} / \mathrm{W}$ rate (Figs 11-12).

Comparing the Somssich Hill 2 Crocidura $M_{1}$ material with the Sima del Elefante TE8-14 (Rofes \& Cuenca-Bescós 2011), Villány 3 and Osztramos 3/2 C. kornfeldi remains (REUMER 1984), the length of the teeth does not show significant differences. On the other hand, the talonids of the Somssich Hill $\mathrm{M}_{1}$ teeth are considerably wider than those of the teeth from the other localities 


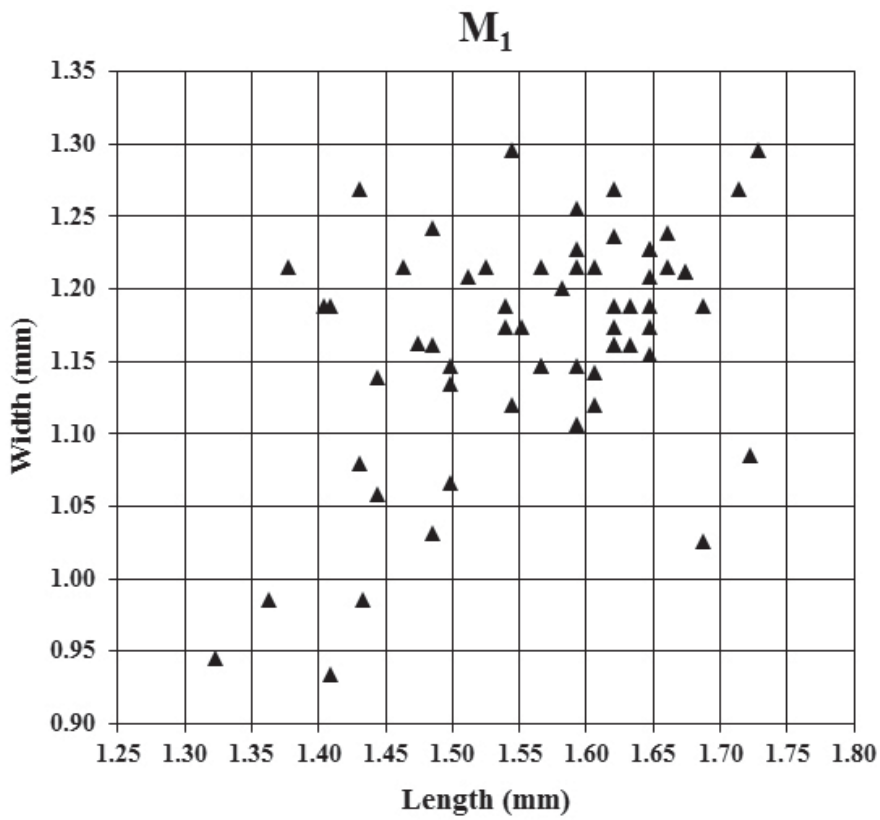

Fig. 10. Scatter plot of the $M_{1} L / W$ rate of the Crocidura remains from the Somssich Hill 2 locality

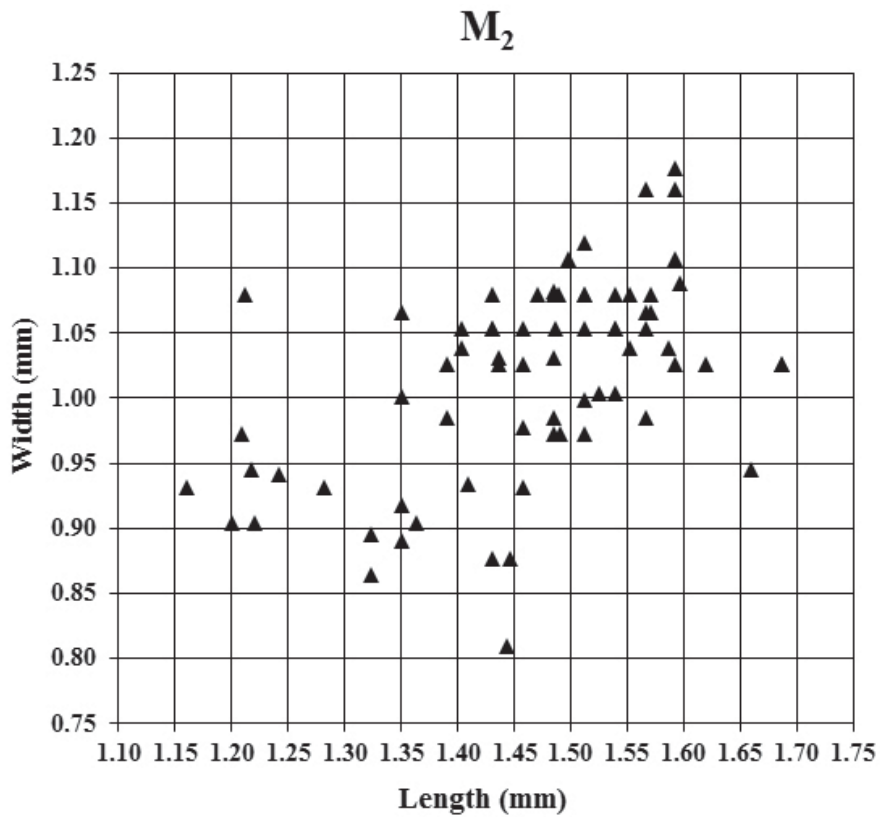

Fig. 11. Scatter plot of the $M_{2} L / W$ rate of the Crocidura remains from the Somssich Hill 2 locality 


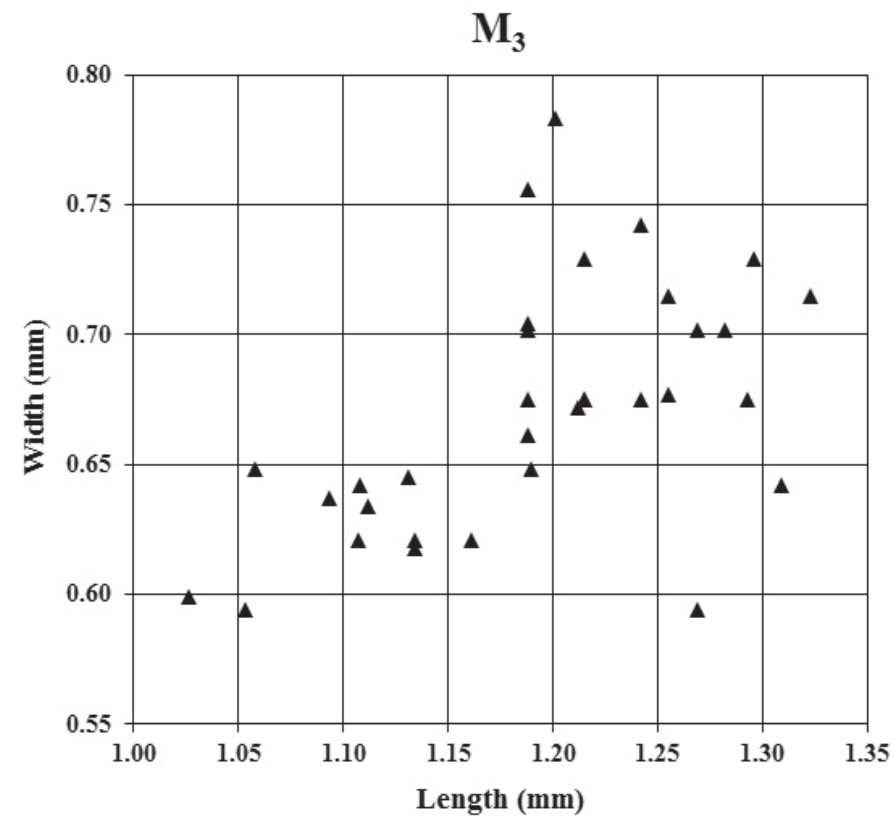

Fig. 12. Scatter plot of the $M_{3} L / W$ rate of the Crocidura remains from the Somssich Hill 2 locality

mentioned. The C. kornfeldi $\mathrm{M}_{1}$ teeth from Betfia (Rzebik-Kowalska 2000) are in the lower range of our data both in length and in width. The dimensions of C. cf. obtusa $\mathrm{M}_{1}$ from Betfia (RzeBiK-Kowalska 2000) perfectly fit to the measurements of the studied specimens (Fig. 13).

\section{CONCLUSIONS}

\section{Biostratigraphy}

The estimated age of the Somssich Hill 2 locality is approximately 1.0-0.9 $\mathrm{Ma}$ (Mimomys savini-M. pusillus biozone by Kordos 1994) on the basis of the vole fauna (Pazonyi et al. 2013a, b. PAzonyi \& Virág 2013a, b, PAzonyi 2015) (Fig. 14). The identified shrew and dormouse fauna (Sorex, Crocidura, Beremendia, Glis, Muscardinus and Dryomimus species) confirmed this hypothesis (BotKa \& STRICZKY 2014, BotKa \& MészÁros 2014a, b, 2015).

C. kornfeldi is first reported in the European mainland at the boundary of the MN16 and MN17 zones from Tourkobounia 3 and 5 localities (Greece, Reumer \& Doukas 1985, Koufos 2001). It disappeared from the continent in the Middle Pleistocene. Its last occurrence is reported in Spain (Cúllar Baza-1, 
Agustí et al. 2010) and in Italy: Rifreddo (MASIni et al. 2005), Tre Fossi and Visogliano, shelter A (KотSAKIs et al. 2003). In Hungary, this is the last (the youngest) appearance of the species (Table 4a).

C. obtusa was present in Central Europe (Austria?, Germany, Hungary, Poland, Romania, and Slovakia), mainly in the Carpathian Basin from the Early Pleistocene (1.2 Ma) to the earliest Late Pleistocene (Biśnik Cave VI, ca. 130-115 ka, Stefania K et al. 2009) (Table 4b).

Summarizing, the age of the site mentioned above is supported by all the Crocidura occurrences reported here.

\section{Palaeoecology}

Crocidurinae prefers milder and more arid climate than the other contemporaneous subfamily (Soricinae) (RzEBIK-Kowalska 1995). According to REUMER (1984), Crocidura prefers dry terrains, hence these forms are good

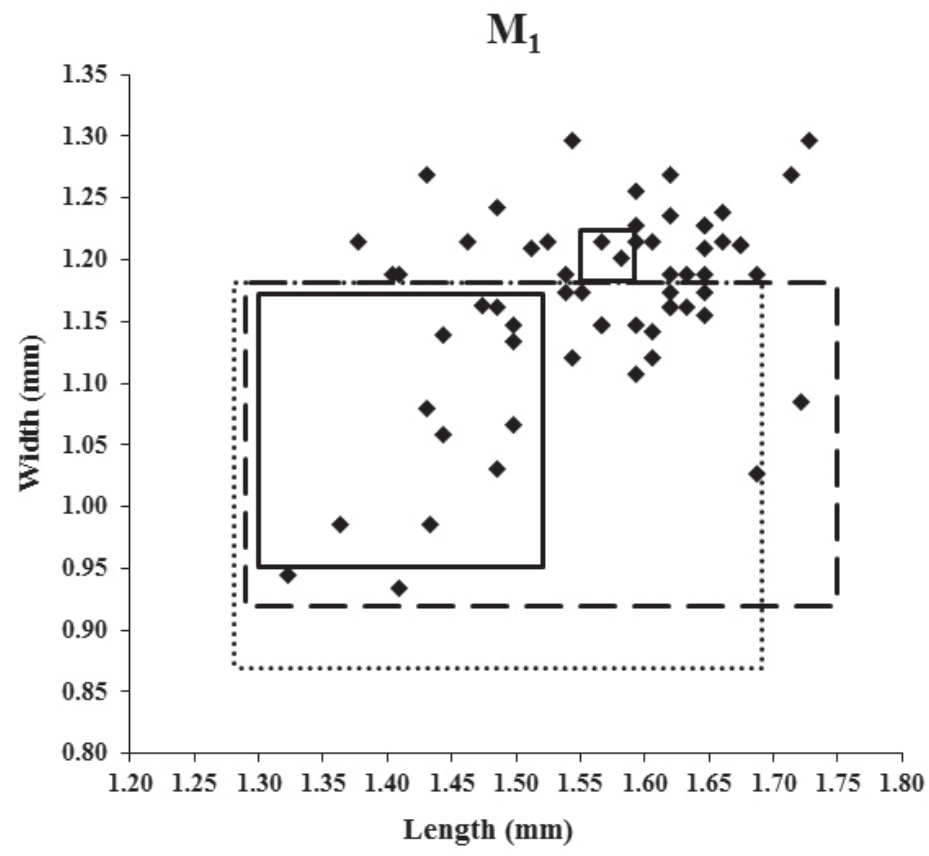

Fig. 13. Scatter plot of the measurements $\left(M_{1} L / W\right.$ rate) of the Crocidura remains from the Somssich Hill 2 locality (marked by black dots), compared with remains from other localities. The frames define the minimum and maximum values of the length and width of $M_{1}$ teeth (small frame with continuous outline $=C$. cf. obtusa from Betfia IX, RzEBIK-KowALS KA 2000; large frame with continuous outline = C. kornfeldi from Betfia V, VII/1, VII/3, IX, X, XI, XII, RZEBIK-KowALSKA 2000; large frame with dashed outline $=C$. kornfeldi from Osztramos 3/2 and Villány 3, REUMER 1984; large frame with dotted outline $=$ C. kornfeldi from Sima del Elefante TE8-14, Rofes \& CuenCA-Bescós 2011) 
Table 4a. The stratigraphical overview of Crocidura kornfeldi and its localities on the basis of the main references. ${ }^{*}=$ The specific identification of these remains is uncertain according to FURIó et al. (2007)

\begin{tabular}{|c|c|c|c|c|c|}
\hline \multirow{2}{*}{$\begin{array}{l}\text { Countries, } \\
\text { Localities }\end{array}$} & \multirow{2}{*}{$\begin{array}{c}\text { Pliocene } \\
\text { MN } 16\end{array}$} & \multicolumn{3}{|c|}{ Pleistocene } & \multirow[t]{2}{*}{ References } \\
\hline & & MN 17 & Early & Middle & \\
\hline Austria & & & & & ZIEGLER \& DAXNER-HöCK (2005) \\
\hline $\begin{array}{l}\text { Deutsch-Alten- } \\
\text { burg } 9,30 \mathrm{~A}\end{array}$ & & + & & & MAIS \& RABEDER $(1977,1984)$ \\
\hline $\begin{array}{l}\text { Deutsch-Alten- } \\
\text { burg 2A, 2C, 4B }\end{array}$ & & & + & & $\begin{array}{l}\text { MAIS \& RABEDER }(1977,1984) \text {, } \\
\text { FRANK \& RABEDER }(1997)\end{array}$ \\
\hline \multicolumn{6}{|l|}{ Croatia } \\
\hline Podumci 1 & & & + & & PAUNOVIĆ \& JAMBREŠIĆ (1997) \\
\hline Razvode & & & + & & PAUNOVIĆ \& JAMBREŠIĆ (1997) \\
\hline Tatinja draga & & & + & & PAUNOVIĆ \& JAMBREŠIĆ (1997) \\
\hline Czech Republic & & & & & FEJFAR \& SABOL (2005) \\
\hline Ctinêves 1 & & + & & & FEJFAR \& HORÁČEK (1983) \\
\hline Greece & & & & & Doukas (2005) \\
\hline $\begin{array}{l}\text { Tourkobounia } \\
3,5\end{array}$ & + & + & & & $\begin{array}{l}\text { REUMER \& Doukas (1985), Kou- } \\
\text { FOS (2001) }\end{array}$ \\
\hline Marathoussa & & & + & & Koufos et al. (2001) \\
\hline Ravin Voulgarakis & & & + & & Koufos (2001) \\
\hline Tourkobounia 2 & & & + & & $\begin{array}{l}\text { REumer \& Doukas (1985), } \\
\text { Koufos (2001) }\end{array}$ \\
\hline \multicolumn{6}{|l|}{ Hungary } \\
\hline Osztramos $3 / 2$ & & cf. & & & $\begin{array}{l}\text { JÁNOSSY \& KoRdos (1977), REU- } \\
\text { MER (1984), JÁNOSSY (1986), MON- } \\
\text { TUIRE (1995), PAZONYi (2011) }\end{array}$ \\
\hline Villány 3 & & + & & & $\begin{array}{l}\text { Reumer (1984), JÁNOSSY (1986), } \\
\text { Montuire (1995), PAZONYi (2011) }\end{array}$ \\
\hline Beremend 16 & & & + & & JÁNossy (1996) \\
\hline $\begin{array}{l}\text { Nagyharsány- } \\
\text { hegy } 2\end{array}$ & & & cf. & & $\begin{array}{l}\text { KREtzoi (1956), Reumer (1984), } \\
\text { JÁNossy (1986) }\end{array}$ \\
\hline $\begin{array}{l}\text { Osztramos 2, } \\
8,14\end{array}$ & & & aff. & & $\begin{array}{l}\text { JÁNOSSY \& KoRdos (1977), REU- } \\
\text { MER (1984), JÁNOSSY (1986), MON- } \\
\text { TUIRE (1995), PAZONYI (2011) }\end{array}$ \\
\hline Somssich Hill 1 & & & + & & REUMER (1984), JÁNOSSY (1986) \\
\hline Somssich Hill 2 & & & + & & $\begin{array}{l}\text { JÁNossy }(1986,1990,1999) \text {, PAzo- } \\
\text { NYI (2011), present article }\end{array}$ \\
\hline Újlak Hill & & & + & & JÁNOSSY (1986), PAZONYI (2011) \\
\hline Villány 5 & & & + & & $\begin{array}{l}\text { Reumer (1984), JÁnossy (1986), } \\
\text { Montuire (1995), PAzonyi (2011) }\end{array}$ \\
\hline
\end{tabular}


Table $4 \mathbf{a}$ (cont.)

\begin{tabular}{|c|c|c|c|c|c|}
\hline \multirow{2}{*}{$\begin{array}{l}\text { Countries, } \\
\text { Localities }\end{array}$} & \multirow{2}{*}{$\begin{array}{c}\text { Pliocene } \\
\text { MN } 16\end{array}$} & \multicolumn{3}{|c|}{ Pleistocene } & \multirow[t]{2}{*}{ References } \\
\hline & & MN 17 & Early & Middle & \\
\hline \multicolumn{6}{|l|}{ Italy } \\
\hline $\begin{array}{l}\text { Montagnola } \\
\text { Senese }\end{array}$ & & + & & & $\begin{array}{l}\text { FANFANI (2000), KoTSAKIs et al. } \\
\text { (2003) }\end{array}$ \\
\hline Pirro Nord & & & + & & $\begin{array}{l}\text { Kotsakis et al. (2003), Petronio } \\
\text { \& Marcolini (2013) }\end{array}$ \\
\hline Rifreddo & & & & + & $\begin{array}{l}\text { KOTSAKIs et al. (2003), SABATO } e t \\
\text { al. (2005), MASINI } e t \text { al. (2005) }\end{array}$ \\
\hline Tre Fossi & & & & + & KotSAKIs et al. (2003) \\
\hline $\begin{array}{l}\text { Visogliano, shel- } \\
\text { ter A }\end{array}$ & & & & + & KоTSAKIs et al. (2003) \\
\hline Poland & & & & & RZEBiK-KowALSKA (2005) \\
\hline Żabia Cave A & & & cf. & & $\begin{array}{l}\text { BosÁK et al. (1982), STEFANIAK et al. } \\
\text { (2009), NADACHOWSKI et al. (2011) }\end{array}$ \\
\hline \multicolumn{6}{|l|}{ Romania } \\
\hline Betfia XIII & & + & & & RZEBIK-KowALSKA $(2000,2002)$ \\
\hline Betfia IX & & & + & & $\begin{array}{l}\text { TERZEA (1994), RzEBIK-Kowals- } \\
\text { KA }(2000,2002)\end{array}$ \\
\hline $\begin{array}{l}\text { Betfia V, VII/1, } \\
\text { VII/3, X, XI, XII }\end{array}$ & & & + & & RZEBIK-KowALSKA $(2000,2002)$ \\
\hline Slovakia & & & & & FejFAR $\&$ Sabol $(2005)$ \\
\hline Pleŝivec & & + & & & FEJFAR \& HORÁČEK (1983) \\
\hline Vĉeláre 3 & & cf. & & & $\begin{array}{l}\text { FEJFAR \& HORÁČEK (1983), HORÁ- } \\
\text { ČEK (1985) }\end{array}$ \\
\hline Spain & & & & & $\begin{array}{l}\text { VAN DEN HOEK OSTENDE \& FURIÓ } \\
\text { (2005) }\end{array}$ \\
\hline $\begin{array}{l}\text { Almenara- } \\
\text { Casablanca } 3\end{array}$ & & & + & & $\begin{array}{l}\text { GIL \& SESÉ (1985), FURIó et al. } \\
\text { (2007), Agustí et al. (2011) }\end{array}$ \\
\hline $\begin{array}{l}\text { Barranco León } \\
\text { 5/D }\end{array}$ & & & + & & $\begin{array}{l}\text { Agustí et al. (2010), CuEnCA-Bes- } \\
\text { cós et al. (2015) }\end{array}$ \\
\hline Cueva Victoria & & & + & & FURIó et al. (2015) \\
\hline Orce 3 & & & $+^{*}$ & & $\begin{array}{l}\text { MARTíN-SUÁREZ (1988), RZEBIK- } \\
\text { KowALSKA (1995), FURIó et al. } \\
\text { (2007) }\end{array}$ \\
\hline $\begin{array}{l}\text { Sima del Elefante } \\
\text { TE7-14 }\end{array}$ & & & + & & $\begin{array}{l}\text { Rofes \& CuenCA-Bescós (2011), } \\
\text { CuencA-Bescós } \text { et al. }(2013,2015) \text {, } \\
\text { Garcia et al. (2014) }\end{array}$ \\
\hline Cúllar Baza-1 & & & & + & Agustí et al. (2010) \\
\hline
\end{tabular}


Table 4b. The stratigraphical overview of Crocidura obtusa and its localities on the basis of the main references

\begin{tabular}{|c|c|c|c|c|c|}
\hline \multirow[t]{2}{*}{ Countries, Localities } & \multicolumn{4}{|c|}{ Pleistocene } & \multirow[t]{2}{*}{ References } \\
\hline & MN 17 & Early & Middle & Late & \\
\hline \multicolumn{6}{|l|}{ Germany } \\
\hline Weißenburg 7 & & + & & & KOENIGSWALD (1971) \\
\hline \multicolumn{6}{|l|}{ Hungary } \\
\hline Beremend 16 & & + & & & JÁNossy (1996) \\
\hline Osztramos 8,14 & & aff. & & & $\begin{array}{l}\text { JÁNOSSY \& KoRdos (1977), JÁNOSSY (1986), } \\
\text { Montuire (1995), PAZONyi (2011) }\end{array}$ \\
\hline Somssich Hill 2 & & + & & & $\begin{array}{l}\text { JÁNOSSY }(1983,1986,1990,1999) \text {, MonTU- } \\
\text { IRE (1995), PAZONYI (2011), present article }\end{array}$ \\
\hline Újlak Hill & & + & & & JÁNossy (1986), PAZONYi (2011) \\
\hline $\begin{array}{l}\text { Castle Hill } \\
\text { (Budapest) }\end{array}$ & & & cf. & & $\begin{array}{l}\text { JÁnossy (1986), Montuire (1995), PAZo- } \\
\text { NYi (2011) }\end{array}$ \\
\hline Kövesvárad & & & cf. & & $\begin{array}{l}\text { JÁnOsSy (1986), Montuire (1995), PAzo- } \\
\text { NYI (2011) }\end{array}$ \\
\hline Nagyharsányhegy 4 & & & + & & $\begin{array}{l}\text { KRetzoi (1956), JÁnossy (1986), Montu- } \\
\text { IRe (1995), PAZONyi (2011) }\end{array}$ \\
\hline Ördöglyuk Cave & & & + & & PAZONYI (2011) \\
\hline Pongor Cave & & & + & & Pazonyi (2011) \\
\hline Tarkő 1, 4, 9-14 & & & cf. & & JÁNossy $(1969,1986)$, Pazonyi (2011) \\
\hline Villány 6 & & & + & & JÁNossy (1986), PAZONYI (2011) \\
\hline Poland & & & & & RZEBIK-KoWALSKA (2005) \\
\hline Przymiłowice 2B & & cf. & & & RZEBIK-KowALSKA (2013) \\
\hline Biśnik Cave VIII, IX & & & + & & STEFANIAK et al. (2009) \\
\hline Biśnik Cave VI & & & & + & STEFANiAK et al. (2009) \\
\hline \multicolumn{6}{|l|}{ Romania } \\
\hline Betfia IX & & cf. & & & RZEBIK-KowALSKA $(2000,2002)$ \\
\hline Ursilor Cave & & cf. & & & RZEBiK-KowALSKa (2002) \\
\hline \multicolumn{6}{|l|}{ Slovakia } \\
\hline Gombasek & & + & & & $\begin{array}{l}\text { KRETZOI }(1938,1941) \text {, WAGNER \& GaSPA- } \\
\text { RIK (2014) }\end{array}$ \\
\hline
\end{tabular}

Fig. 14. Stratigraphical position of the Somssich Hill 2 locality (after Kordos 1994, KRETZOr 1969, KRETZOI \& PÉCsi 1982, PAzonyi et al. 2013a), and the stratigraphical range of the studied species (CB = Carpathian Basin, ${ }^{*}=$ after KRETZOI 1969, KRETZOI \& PÉCSI 1982, Nhh. = Nagyharsányhegy

Phase, Th. $=$ Templomhegy Phase, T.-V. $=$ Tarkő-Vértesszőlős Phase and ${ }^{* *}=$ Uppony Phase $)$ 


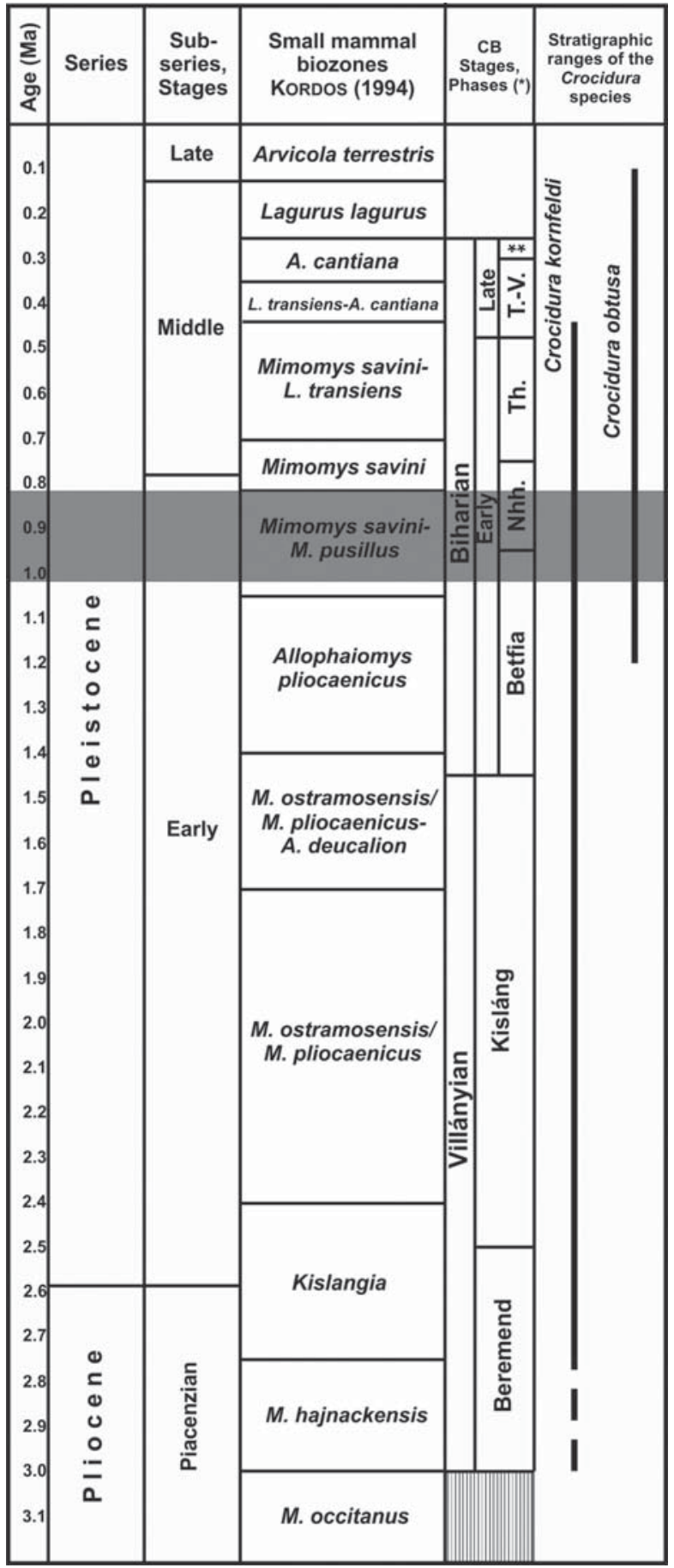


indicators of more or less open grasslands in the warmer phases of the PlioPleistocene (RzebiK-KowalsKa 1995).

The separation of the two Crocidura species is difficult because of the morphological similarities in the succession of the Somssich Hill 2 locality. However, the occurrence of the genus is very useful as a palaeoecological indicator. Its abundance can be evaluated only in comparison with the Sorex remains. The

Table 5. The occurrence of the genus Crocidura in the layers of the Somssich Hill 2 locality, with the number of specimens, the number of teeth, and the minimum number of individuals (MNI)

\begin{tabular}{|c|c|c|c|c|c|c|c|}
\hline Layer & $\begin{array}{l}\text { Number of } \\
\text { specimens }\end{array}$ & $\begin{array}{l}\text { Number } \\
\text { of teeth }\end{array}$ & MNI & Layer & $\begin{array}{l}\text { Number of } \\
\text { specimens }\end{array}$ & $\begin{array}{l}\text { Number } \\
\text { of teeth }\end{array}$ & MNI \\
\hline 1 & - & - & - & 26 & 3 & 4 & 1 \\
\hline 2 & - & - & - & 27 & - & - & - \\
\hline 3 & - & - & - & 28 & - & - & - \\
\hline 4 & 30 & 33 & 5 & 29 & 4 & 4 & 3 \\
\hline 5 & 56 & 97 & 11 & 30 & 3 & 6 & 3 \\
\hline 6 & 12 & 19 & 3 & 31 & 4 & 6 & 2 \\
\hline 7 & 3 & 4 & 2 & 32 & - & - & - \\
\hline 8 & 9 & 24 & 3 & 33 & 2 & 4 & 1 \\
\hline 9 & 4 & 10 & 1 & 34 & - & - & - \\
\hline 10 & 14 & 32 & 4 & 35 & - & - & - \\
\hline 11 & 6 & 15 & 3 & 36 & 1 & 1 & 1 \\
\hline 12 & 9 & 29 & 3 & 37 & 3 & 5 & 1 \\
\hline 13 & 8 & 13 & 3 & 38 & 6 & 7 & 2 \\
\hline 14 & 5 & 12 & 3 & 39 & 1 & - & 1 \\
\hline 15 & 1 & 2 & 1 & 40 & - & - & - \\
\hline 16 & 3 & 6 & 2 & 41 & 2 & 4 & 2 \\
\hline 17 & - & - & - & 42 & 1 & 1 & 1 \\
\hline 18 & 1 & 4 & 1 & 43 & 1 & 1 & 1 \\
\hline 19 & - & - & - & 44 & 1 & 4 & 1 \\
\hline 20 & 1 & 3 & 1 & 45 & 2 & 4 & 1 \\
\hline 21 & - & - & - & 46 & 3 & 4 & 2 \\
\hline 22 & 4 & 6 & 2 & 47 & - & - & - \\
\hline 23 & - & - & - & 48 & - & - & - \\
\hline 24 & 1 & 2 & 1 & 49 & - & - & - \\
\hline \multirow[t]{2}{*}{25} & 4 & 9 & 4 & 50 & - & - & - \\
\hline & & & & $\Sigma$ & 208 & 375 & 76 \\
\hline
\end{tabular}


study of Sorex species is currently in progress, the results will be published in the next volume of this journal. However, some conclusions are deduced merely on the basis of the Crocidura species.

The crocidurines are completely absent in the lowermost layers of the succession (layers 50-47). This fact suggests the presence of a forested environment. After this period open grasslands were also present beside the closed vegetation in most part of the time interval represented by the layers $46-4$. The occurrence of the genus was the most frequent in the layers 16-4. They are absent in the uppermost layers (layers 3-1) as well as in the lowermost part of the sequence. The absence of Crocidura species in the uppermost layers is not an evidence for the disappearance of the open grassy vegetation, because the small number of specimens makes the evaluations uncertain (Table 5).

Acknowledgements - The work was supported by the Hungarian Scientific Research Fund (OTKA K104506 project). The authors are indebted to the members of the OTKA Research Team, mainly to Piroska Pazonyi (project leader), Zoltán Szentesi, Mihály Gasparik and Attila Virág for their useful help and valuable suggestions. Special thanks to Károly Bóka for his kind help in making the SEM photos and to Piroska Pazonyi for her useful reviewer comments.

\section{REFERENCES}

Agustí J., Blain H.-A., Furió M., De Marfá R. \& Santos-Cubedo A. 2010: The early Pleistocene small vertebrate succession from the Orce region (Guadix-Baza Basin, SE Spain) and its bearing on the first human occupation of Europe. - 2uaternary International 223: 162-169. http://dx.doi.org/10.1016/j.quaint.2009.12.011

Agustí J., Santos-Cubedo A., Furió M., De Marfá R., Blain H.-A., Oms O. \& Sevilla P. 2011: The late Neogene-early Quaternary small vertebrate succession from the AlmenaraCasablanca karst complex (Castellón, Eastern Spain): Chronologic and paleoclimatic context. - 2uaternary International 243: 183-191. http://dx.doi.org/10.1016/j.quaint.2010.11.016

Bosák P., Glazek J., Horáček I. \& Szynkiewicz A. 1982: New locality of Early Pleistocene vertebrates - Żabia Cave at Podlesice, Central Poland. - Acta Geologica Polonica 32: 217-226.

Bot KA D. \& MÉszÁros L. 2014a: Beremendia (Mammalia, Soricidae) remains from the late Early Pleistocene Somssich Hill 2 locality (Southern Hungary) and their taxonomic, biostratigraphical, palaeoecological and palaeobiogeographical relations. - Fragmenta Palaeontologica Hungarica 31: 83-115. http://dx.doi.org/10.17111/FragmPalHung.2014.31.83

BotкA D. \& MÉszÁros L. 2014b: A Somssich-hegy 2-es lelőhely alsó-pleisztocén Soricidae faunája. [The Lower Pleistocene Soricidae fauna of the Somssich Hill 2 locality.] - In: BosNA KOFF M. \& Dulai A. (eds): Program, Elöadáskivonatok, Kirándulásvezetö, 17. Magyar Öslénytani Vándorgyülés, Győr, pp. 10-11. (in Hungarian)

BotKA D. \& MÉszáros L. 2015: A Somssich-hegy 2-es lelőhely (Villányi-hegység) alsó-pleisztocén Beremendia fissidens (Mammalia, Soricidae) maradványainak taxonómiai és paleoökológiai vizsgálata. (Taxonomic and palaeoecological studies on the Lower Pleistocene Beremendia fissidens (Mammalia, Soricidae) remains of the Somssich Hill 2 locality (Villány Hills).) - Földtani Közlöny 145(1): 73-84. 
Bot KA D. \& STRICZ KY L. 2014: Soricidae and Gliridae fauna of the late Early Pleistocene Somssich Hill 2 locality (South Hungary). - Acta Mineralogica-Petrographica Abstract Series 8: 12.

Cuenca-Bescós G., Blain H.-A., Rofes J., Lozano-Fernández I., López-García J. M., Duval M., Galán J. \& NúŃEZ-Lahuerta C. 2015: Comparing two different Early Pleistocene microfaunal sequences from the caves of Atapuerca, Sima del Elefante and Gran Dolina (Spain): biochronological implications and significance of the Jaramillo subchron. - 2uaternary International 389: 148-158. http://dx.doi.org/10.1016/j.quaint.2014.12.059

Cuenca-Bescós G., Rofes J., López-García J. M., Blain H.-A., Rabal-Garcés R., Saulué V., Arsuaga J. L., Bermúdez de Castro J. M. \& Carbonell E. 2013: The small mammals of Sima del Elefante (Atapuerca, Spain) and the first entrance of Homo in Western Europe. 2uaternary International 295: 28-35. http://dx.doi.org/10.1016/j.quaint.2011.12.012

Doukas C. S. 2005: Greece. - In: Hoek Ostende L. W. van den, Doukas C. S. \& Reumer J. W. F. (eds): The fossil record of the Eurasian Neogene insectivores (Erinaceomorpha, Soricomorpha, Mammalia), Part I. - Scripta Geologica Special Issue 5: 99-112.

FANFANI F. 2000: Revisione degli insettivori (Mammalia) tardo neogenici e quaternari dell'Italia peninsulare. [Revision of the insectivores (Mammalia) from the Late Neogene and Quaternary of the Italian Peninsula.] - Unpublished PhD thesis, University of Bologna-Firenze-ModenaRoma 'La Sapienza', 282 pp.

Fejfar O. \& HoráČEK I. 1983: Zur Entwicklung der Kleinsäugerfaunen im Villányium und AltBiharium auf dem Gebiet der ČSSR. - Schriftenreihe für Geologische Wissenschaften 19-20: 111-207.

Fejfar O. \& Sabol M. 2005: Czech Republic and Slovak Republic. - In: Hoek Ostende L. W. van Den, Doukas C. S. \& Reumer J. W. F. (eds): The fossil record of the Eurasian Neogene insectivores (Erinaceomorpha, Soricomorpha, Mammalia), Part I. - Scripta Geologica Special Issue 5: 51-60.

Fran K C. \& Rabeder G. 1997: Deutsch-Altenburg. - In: Döppes D. \& Rabeder G. (eds): Pliozäne und pleistozäne Faunen Österreichs. - Mitteilungen der Kommission für 2uartärforschung der Österreichischen Akademie der Wissenschaften 10: 270-274.

Furió M., Gibert L., Ferràndez C. \& Sevilla P. 2015: The insectivores (Soricidae, Erinaceidae; Eulipotyphla; Mammalia) from Cueva Victoria (Early Pleistocene, Murcia, Spain). - Neues Jahrbuch für Geologie und Paläontologie Abhandlungen 275(2): 151-161. http://dx.doi.org/10.1127/njgpa/2015/0460

Furió M., Santos-Cubedo A., Minwer-Barakat R. \& Agustí J. 2007: Evolutionary history of the African soricid Myosorex (Insectivora, Mammalia) out of Africa. - Journal of Vertebrate Paleontology 27(4): 1018-1032. http://dx.doi.org/10.1671/0272-4634(2007)27[1018:EHOTAS]2.0.CO;2

Garcia J., Martínez K., Cuenca-Bescós G. \& Carbonell E. 2014: Human occupation of Iberia prior to the Jaramillo magnetochron (>1.07 Myr). - Quaternary Science Reviews 98: 84-99. http://dx.doi.org/10.1016/j.quascirev.2014.05.031

GiL E. \& SESÉ C. 1985: Micromamíferos (Insectivora, Rodentia y Lagomorpha) del nuevo yacimiento villafranquiense de Casablanca B (Almenara, prov. de Castellón). - Estudios geológicos 41: 495-501.

HíR J. 1998: Cricetids (Rodentia, Mammalia) of the Early Pleistocene vertebrate fauna of Somssich Hill 2 (Southern Hungary, Villány Mountains). - Annales historico-naturales Musei nationalis hungarici 90: 57-89.

Hoek Ostende L. W. van den \& Furió M. 2005: Spain. - In: Hoek Ostende L. W. van den, Doukas C. S. \& Reumer J. W. F. (eds): The fossil record of the Eurasian Neogene insecti- 
vores (Erinaceomorpha, Soricomorpha, Mammalia), Part I. - Scripta Geologica Special Issue 5: 149-284.

HORÁČEK I. 1985: Survey of the fossil vertebrate localities Včeláre 1-7. - Časopis pro mineralogii a geologii 30(4): 353-366.

JÁnossy D. 1969: Stratigraphische Auswertung der europäischen mittelpleistozänen Wirbeltierfauna. Teile I.-II. - Berichte der Deutschen Gesellschaft für Geologische Wissenschaften. Reihe A, Geologie und Paläontologie 14(4-5): 367-438, 573-643.

JÁnossy D. 1983: Lemming-remain from the Older Pleistocene of Southern Hungary (Villány, Somssich Hill 2). - Fragmenta Mineralogica et Palaeontologica 11: 55-60.

JÁnossy D. 1986: Pleistocene Vertebrate Faunas of Hungary. - Akadémiai Kiadó, Budapest, 208 pp.

JÁnossy D. 1990: Arvicolids from the Lower Pleistocene sites at Beremend 15 and Somssich-hegy 2, Hungary. - In: FEJFAR O. \& HEINRICH W. D. (eds): International Symposium of Evolution, Phylogeny and Biostratigraphy of Arvicolids, pp. 223-230.

JÁnossy D. 1996: Lower Pleistocene vertebrate faunas from the localities 16 and 17 of Beremend (Southern Hungary). - Fragmenta Mineralogica et Palaeontologica 18: 91-102.

JÁnossy D. 1999: Újabb adatok a villányi Somssich-hegy 2. lelőhely leleteihez. [New data on the Somssich Hill 2 locality, Villány.] - Manuscript, Hungarian Natural History Museum, Budapest, pp. 1-10. (in Hungarian)

JÁnossy D. \& Kordos L. 1977: Az Osztramos gerinces lelőhelyeinek faunisztikai és karsztmorfológiai áttekintése (1975-ig). [Faunistical and karstmorphological review of palaeontological localities for vertebrates at Osztramos (Northern Hungary).] - Fragmenta Mineralogica et Palaeontologica 8: 39-72.

Koenigswald W. V. von 1971: Die altpleistozäne Wirbeltierfaunula aus der Spaltenfüllung Weissenburg 7 (Bayern). - Mitteilungen der Bayerischen Staatssammlung für Paläontologie und Historische Geologie 11: 117-122.

Kordos L. 1994: Revised Biostratigraphy of the Early Man Site at Vértesszőlős, Hungary. - Courier Forschungs-Institut Senckenberg 171: 225-236.

Kormos T. 1934: Neue Insektenfresser, Fledermäuse und Nager aus dem Oberpliozän der Villányer Gegend. - Földtani Közlöny 64(1): 296-321.

Kormos T. 1937: Zur Geschichte und Geologie der oberpliozänen Knochenbreccien des Villányer Gebirges. - Matematikai és Természettudományi Értesitö 56: 1061-1110.

Kotsakis T., Abbazzi L., Angelone C., Argenti P., Barisone G., Fanfani F., Marcolini F. \& MASINi F. 2003: Plio-Pleistocene biogeography of Italian mainland micromammals. - In: Reumer J. W. F. \& Wessels W. (eds): Distribution and Migration of Tertiary Mammals in Eurasia. A Volume in Honour of Hans De Bruijn. - Deinsea 10: 313-342.

Koufos G. D. 2001: The Villafranchian mammalian faunas and biochronology of Greece. - Bollettino della Società Paleontologica Italiana 40(2): 217-223.

Koufos G. D., Vassiliadou K. V., Koliadimou K. K. \& Syrides G. E. 2001: Early Pleistocene small mammals from Marathoussa, a new locality in the Mygdonia basin, Macedonia, Greece. - Deinsea 8: 49-102.

Kretzoi M. 1938: Die Raubtiere von Gombaszög nebst einer übersicht der Gesamtfauna (Ein Beitrag zur stratigraphie des Altquartaers). - Annales historico-naturales Musei nationalis hungarici 31: 88-157.

Kretzoi M. 1941: Weitere Beiträge zur Kenntnis der Fauna von Gombaszög. - Annales historiconaturales Musei nationalis hungarici 34: 105-139.

Kretzoi M. 1956: Die Altpleistozänen Wirbeltierfaunen des Villányer Gebirges. - Geologica Hungarica, Series Palaeontologica 27: 1-264. 
KRETzor M. 1969: A magyarországi quarter és pliocén szárazföldi biosztratigráfiájának vázlata. [The sketch of the Quaternary and Pliocene terrestrial biostratigraphy of Hungary.] Földrajzi Közlemények 17: 179-204. (in Hungarian)

Kretzoi M. \& PÉCsi M. 1982: A Pannóniai-medence pliocén és pleisztocén időszakának tagolása. [The Pliocene and Pleistocene stratigraphy of the Pannonian Basin.] - Földrajzi Közlemények 30(4): 300-326. (in Hungarian)

Krolopp E. 2000: Alsó-pleisztocén Mollusca-fauna a Villányi-hegységből. [Lower Pleistocene mollusc fauna from the Villány Mts. (Southern Hungary).] - Malakológiai Tájékoztató 18: 51-58. (in Hungarian, with English abstract)

Mais K. \& RABEDER G. 1977: Eine pliozäne Höhlenfüllung im Pfaffenberg bei Bad DeutschAltenburg (Niederösterreich). - Die Höhle 28(1): 1-7.

MAIs K. \& RABEDER G. 1984: Das größe Höhlensystem im Pfaffenberg bei Bad Deutsch-Altenburg (Niederösterreich) und seine fossilen Faunen. - Die Höhle 35(3-4): 213-230.

Martín-SuÁrez E. 1988: Sucesiones de micromamíferos en la depresión Guadix-Baza (Granada, España). [The small mammal assemblages of the Guadix-Baza Basin (Granada, Spain).] Unpublished $\mathrm{PhD}$ thesis, Universidad de Granada, $241 \mathrm{pp}$.

Masini F., Giannini T., Abbazzi L., Fanfani F., Delfino M., Maul L. C. \& Torre D. 2005: A latest Biharian small vertebrate fauna from the lacustrine succession of San Lorenzo (Sant'Arcangelo Basin, Basilicata, Italy). - 2uaternary International 131(1): 79-93. http://dx.doi.org/10.1016/j.quaint.2004.07.008

MÉSzÁros L. 2015: Öslénytani kutatás és tehetséggondozás: Elözetes jelentés az alsó-pleisztocén Somssich-hegy 2-es lelőhely Sorex-Crocidura fog-arány vizsgálatáról. [Preliminary report on the study of the Sorex-Crocidura (Mammalia, Soricidae) relation in the Lower Pleistocene fossil assemblage of the Somssich Hill 2 locality (Hungary).] - Acta Pintériana 1: 15-24. (in Hungarian)

Montuire S. 1995: Évolution climatique et diversité chez les mammifères en Europe centrale depuis le Pliocène. - Geobios 28: 313-327. http://dx.doi.org/10.1016/S0016-6995(95)80177-4

Nadachowski A., Stefaniak K., Szynkiewicz A., Marciszak A., Socha P., SChick P. \& AUgust C. 2011: Biostratigraphic importance of the Early Pleistocene fauna from Żabia Cave (Poland) in Central Europe. - 2uaternary International 243(1): 204-218. http://dx.doi.org/10.1016/j.quaint.2011.04.037

Pálfy J., Dulai A., Gasparik M., Ozsvárt P., Pazonyi P. \& Szives O. 2008: Catalogue of invertebrate and vertebrate paleontological type specimens of the Hungarian Natural History Museum. - Hungarian Natural History Museum, Budapest, 209 pp.

PAunović M. \& JAMBREšić G. 1997: Review of the Results of Morphometric and Morphogenetic Analyses of Early Pleistocene Micromammals and Upper Pleistocene Cave Bears in Croatia. - Geologia Croatica 50(2): 225-230.

Pazony P. 2011: Palaeoecology of Late Pliocene and Quaternary mammalian communities in the Carpathian Basin. - Acta Zoologica Cracoviensia 54A(1-2): 1-29. http://dx.doi.org/10.3409/azc.54a_1-2.01-29

Pazonyi P. 2015: A Somssich-hegyi óriáspocok fauna. [The giant vole fauna of the Somssich Hill.] - In: Bosna koff M. \& Dulai A. (eds): Program, Elöadáskivonatok, Kirándulásvezetö, 18. Magyar Öslénytani Vándorgyülés, Varbó-Fónagyság, pp. 27-28.

Pazonyi P., Mészáros L., Szentesi Z., Gasparik M. \& Virág A. 2013a: Preliminary results of the palaeontological investigations of the late Early Pleistocene Somssich Hill 2 locality (South Hungary). - In: CAGATAY N. \& ZABCI C. (eds): Book of Abstracts of the RCMNS 2013, Istanbul Technical University, p. 270. 
Pazonyi P., Mészáros L., Szentesi Z., Gasparik M. \& Virág A. 2013b: A Somssich-hegy 2-es lelőhely gerinces faunájának új kutatási eredményei. [New results of the palaeontological investigation of the Somssich Hill 2 vertebrate fauna.] - In: Bosnakof F M., Dulai A., Vörös A. \& PÁlfy J. (eds): Program, Elöadáskivonatok, Kirándulásvezetö, 16. Magyar Öslénytani Vándorgyülés, Orfü, pp. 30-31.

Pazonyi P. \& Virág A. 2013a: A Microtus genus (Mammalia, Arvicolinae) landmark elemzése a Somssich-hegy 2-es lelöhelyről. [Landmark analysis of the genus Microtus (Mammalia, Arvicolinae) from the Somssich Hill 2 locality.] - In: BosNAkoff M., Dulai A., Vörös A. \& PÁlfy J. (eds): Program, Elöadáskivonatok, Kirándulásvezetö, 16. Magyar Öslénytani Vándorgyülés, Orfü, pp. 32-33.

PAzonyi P. \& Virág A. 2013b: Landmark analysis of first lower molars of genus Microtus from the late Early Pleistocene Somssich Hill 2 locality (South Hungary) and its evolutionary implications. - Systematics, phylogeny and palaeontology of small mammals, St. Petersburg, 11-14 November 2013, p. 10.

Petronio C. \& Marcolini F. 2013: Mammal Biochronology at the end of Late Villafranchian (Early Pleistocene): Pirro Faunal Unit. - Palaeontographica Abteilung A: Palaeozoology - Stratigraphy 298(1-6): 183-191.

Repenning C. A. 1967: Subfamilies and genera of the Soricidae. - Geological Survey Professional Paper 565: 1-74.

Reumer J. W. F. 1984: Ruscinian and early Pleistocene Soricidae (Insectivora, Mammalia) from Tegelen (The Netherlands) and Hungary. - Scripta Geologica 73: 1-173.

Reumer J. W. F. \& Doukas C. S. 1985: Early Pleistocene Insectivora (Mammalia) from Tourkobounia (Athens, Greece). - Proceedings Koninklijke Nederlandse Akademie van Wetenschappen B88(1): 111-121.

Rofes J. \& CuenCA-Bescós G. 2011: Evolutionary history and biogeography of the genus Crocidura (Mammalia, Soricidae) in Europe, with emphasis on Crocidura kornfeldi. - Mammalian Biology 76(1): 64-78. http://dx.doi.org/10.1016/j.mambio.2009.12.001

RzeBiK-Kowals KA B. 1995: Climate and history of European shrews (family Soricidae). - Acta Zoologica Cracoviensia 38(1): 95-107.

RZEBIK-KowAls KA B. 2000: Insectivora (Mammalia) from the Early and early Middle Pleistocene of Betfia in Romania. I. Soricidae Fischer von Waldheim, 1817. - Acta Zoologica Cracoviensia 43(1-2): 1-53.

Rzebik-Kowals Ka B. 2002: The Pliocene and Early Pleistocene Lipotyphla (Insectivora, Mammalia) from Romania. - Acta Zoologica Cracoviensia 45(2): 251-281.

Rzebik-Kowalska B. 2005: Poland. - In: Hoek Ostende L. W. van den, Doukas C. S. \& REUMER J. W. F. (eds): The Fossil Record of the Eurasian Neogene insectivores (Erinaceomorpha, Soricomorpha, Mammalia), Part I. - Scripta Geologica Special Issue 5: 119-134.

RzEBIK-KowALs KA B. 2013: Sorex bifidus n. sp. and the rich insectivore mammal fauna (Erinaceomorpha, Soricomorpha, Mammalia) from the Early Pleistocene of Żabia Cave in Poland. Palaeontologia Electronica 16(2/12A): 1-35.

Sabato L., Bertini A., Masini F., Albianelli A., Napoleone G. \& Pieri P. 2005: The lower and middle Pleistocene geological record of the San Lorenzo lacustrine succession in the Sant'Arcangelo Basin (Southern Apennines, Italy). - Quaternary International 131(1): 59-69. http://dx.doi.org/10.1016/j.quaint.2004.07.001

Stefaniak K., Tyc A. \& Socha P. (eds) 2009: Karst of the Częstochowa Upland and of the Eastern Sudetes: palaeoenvironments and protection. - Studies of the Faculty of Earth Sciences University of Silesia, Wroclaw, $535 \mathrm{pp}$. 
Striczky L. \& Pazonyi P. 2014: Taxonomic study of the dormice (Gliridae, Mammalia) fauna from the late Early Pleistocene Somssich Hill 2 locality (Villány Hills, South Hungary) and its palaeoecological implications. - Fragmenta Palaeontologica Hungarica 31: 51-81. http://dx.doi.org/10.17111/FragmPalHung.2014.31.51

SZENTESI Z. 2014: Előzetes eredmények a késői kora-pleisztocén Somssich-hegy 2 (Villányi-hegység) ősgerinces-lelőhely kétéltűinek vizsgálatában. (Preliminary results on a study of amphibians of the late Early Pleistocene Somssich Hill 2 palaeovertebrate locality (Villány Mountains).) - Földtani Közlöny 144(2): 165-174.

TERzEA E. 1994: Fossiliferous sites and the chronology of mammal faunas at Betfia (Bihor, Romania). - Travaux du Museum National d'Histoire Naturelle "Grigore Antipa" 34: 467-485.

WAGNER J. \& GASPARIK M. 2014: Research history of Pleistocene faunas in Gombasek quarry (Slovakia), with comments to the type specimen and the type locality of Ursus deningeri gombaszogensis Kretzoi, 1938. - Fragmenta Palaeontologica Hungarica 31: 125-143.

http://dx.doi.org/10.17111/FragmPalHung.2014.31.125

Ziegler R. \& Daxner-Höck G. 2005: Austria. - In: Hoek Ostende L. W. van den, Doukas C. S. \& Reumer J. W. F. (eds): The fossil record of the Eurasian Neogene insectivores (Erinaceomorpha, Soricomorpha, Mammalia), Part I. - Scripta Geologica Special Issue 5: 11-29. 